\title{
Design of Efficient Off-Grid Solar Photovoltaic Water Pumping System Based on Improved Fractional Open Circuit Voltage MPPT Technique
}

\author{
Ali Hmidet $\left(\mathbb{D},{ }^{1}\right.$ Umashankar Subramaniam, ${ }^{2}$ Rajvikram Madurai Elavarasan, ${ }^{3}$ \\ Kannadasan Raju, ${ }^{4}$ Matias Diaz, ${ }^{5}$ Narottam Das ${ }^{6},{ }^{6,7}$ Kashif Mehmood, ${ }^{8}$ \\ Alagar Karthick $\left({ }^{\circ},{ }^{9}\right.$ M. Muhibbullah $\left(\mathbb{1},{ }^{10}\right.$ and Olfa Boubaker $\mathbb{(}^{11}$ \\ ${ }^{1}$ University of Tunis El Manar, Higher Institute of Medical Technologies of Tunis (ISTMT), Tunisia \\ ${ }^{2}$ Renewable Energy Lab, Department of Communications and Networks, Prince Sultan University, Saudi Arabia \\ ${ }^{3}$ Department of Electrical and Electronics Engineering, Thiagarajar College of Engineering, Madurai 625015, Tamilnadu, India \\ ${ }^{4}$ Department of Electrical and Electronics Engineering, Sri Venkateswara College of Engineering, Sriperumbudur, Tamilnadu, India \\ ${ }^{5}$ University of Santiago of Chile, Electrical Engineering Department, Santiago, Chile \\ ${ }^{6}$ School of Engineering and Technology, Central Queensland University, Melbourne, VIC 3000, Australia \\ ${ }^{7}$ Centre for Intelligent Systems, School of Engineering and Technology, Central Queensland University, Brisbane, \\ QLD 4000, Australia \\ ${ }^{8}$ School of Electrical Engineering, Southeast University, Nanjing, China \\ ${ }^{9}$ Renewable Energy Lab, Department of Electrical and Electronics Engineering, KPR Institute of Engineering and Technology, \\ Arasur Coimbatore, 641407 Tamilnadu, India \\ ${ }^{10}$ Department of Electrical and Electronic Engineering, Bangladesh University, Dhaka 1207, Bangladesh \\ ${ }^{11}$ University of Carthage, National Institute of Applied Sciences and Technology, Tunis, Tunisia \\ Correspondence should be addressed to M. Muhibbullah; m.muhibbullah@bu.edu.bd
}

Received 26 July 2021; Revised 13 September 2021; Accepted 14 September 2021; Published 14 October 2021

Academic Editor: Laurentiu Fara

Copyright (C) 2021 Ali Hmidet et al. This is an open access article distributed under the Creative Commons Attribution License, which permits unrestricted use, distribution, and reproduction in any medium, provided the original work is properly cited.

\begin{abstract}
The main application of off-grid solar photovoltaic (SPV) systems is water extraction in rural areas where access to the grid is restricted. In this application, photovoltaic (PV) and pump system regulation are crucial to increase its overall efficiency. In this context, this work presents a simple and efficient off-grid SPV water pumping system (SPVWPS). The designed system is based on a DC-DC boost converter, a three-phase DC-AC inverter, and a three-phase induction motor (IM) coupled to the centrifugal pump. The proposed solution is operated using a control strategy that associates an improved fractional open-circuit voltage (FOCV) method for maximum power point tracking (MPPT) and closed-loop scalar control. This association avoids the use of a speed sensor/encoder and a current sensor for the IM. Finally, the effectiveness of the proposed off-grid SPVWPS and its control system for both steady-state and dynamic conditions of insolation change is verified using a 1KVA rated prototype. The relevance of the drive is also checked in various operating conditions and is found to be adequate for pumping water. Moreover, the proposed method guarantees a fast response, less oscillations around the MPP, a system efficiency of $99 \%$, and a high flow rate due to the extraction of maximum power.
\end{abstract}

\section{Introduction}

The generation based on fossil fuels from coal and oil threatens climatic conditions, accelerating carbon emissions [1]. In developing countries, the capacity to mount solar photovoltaic (PV) panels has increased significantly for more than a decade due to volatility in oil prices [2]. However, the applications of energy using solar photovoltaic (SPV) generators can be varied because of their enormous benefits. The SPV isolated systems are low price, secure, 
and straightforward solutions for decentralised energy supply. They allow secure and decentralised electrical sources to be installed in areas far away from power plants. The isolated PV systems are used in telecommunications, rural electrification, agricultural, street lighting, signage, control, and rural development. One of the most critical applications of PV systems in agriculture is the water pumping system. Such an application makes it possible to extract water in rural areas, where the cost of installing a conventional line is too high [3-6]. In [7], the authors proposed a fuzzy precompensated hybrid proportional integral controller for permanent magnet motor driven solar water pumping system that overcomes conventional controller shortcomings. In [8], the authors proposed indirect field-oriented control for IM without an energy storage system to improve the performance of the PV water pumping system. The gridconnected motor-driven solar-powered water pumping system with efficient control is proposed in [9], which provides MPP tracking along with bidirectional power flow between the grid and PV.

Different kinds of IM control theories have been tested and validated in recent years to boost performance. Among these control methods, scalar control, known as constant stator flux $V / f$ control, field-oriented control (FOC), and direct torque control (DTC), is significant [10-12]. Scalar control is the most popular form of variable speed drive for its low cost, ease of use, and applications that do not require high regulation at low speeds $[13,14]$. The purpose of this technique is achieved by the control of the stator voltage and frequency to conserve the flux at a constant value. Unlike FOC and DTC, this technique does not require extra current sensors.

Since the SPV-based water pumping system is well affected by variable climatic conditions, there is always a need to optimize the energy utilisation. The PV source should always be exploited at its maximum efficiency point, i.e., maximum power point (MPP). Therefore, a convenient MPP Tracking (MPPT) scheme is required. A complete review of MPPT for off-grid SPV systems can be discovered in the literature, which comprises a constant voltage (CV), the fractional open-circuit voltage (FOCV), the fractional short circuit current (FSCC), the perturb and observe $(\mathrm{P} \& \mathrm{O})$, the incremental conductance (IC), artificial neural network (ANN), fuzzy logic control (FLC), genetic algorithm (GA), and upgraded $\mathrm{P} \& \mathrm{O}$ based techniques [15-28].

In general, these techniques are classified into two categories: direct and indirect techniques [17, 25]. Direct MPPT techniques incorporate methods based on the PV measurement of current and voltage, i.e., P\&O, IC, ANN, and FLC methods. On the other hand, the indirect methods use a database including empirical data or mathematical relationships resulting in corrections and approximations to estimate the MPP, i.e., CV, FSCC, and FOCV. The FOCV control is based on a proportional relation between the optimal voltage and the characteristic parameters of the PV module that is the open-circuit voltage $[15,16,19,21,26]$. This MPPT technique requires only one sensor for validation. Therefore, it is less expensive than $\mathrm{P} \& \mathrm{O}$ and IC methods. Nevertheless, from the point of view of precision, it is less efficient compared to P\&O control due to the estimation of the open-circuit voltage parameter $\left(V_{\text {oC }}\right)$ and the difficulty of interrupting the conversion to take measurements too often of $V_{\mathrm{OC}}$. So, the algorithm of this command is slower than of the $\mathrm{P} \& \mathrm{O}$ method $[17,27]$.

Generally, the control algorithms for PV and other applications are implemented using microcontrollers to reduce the size and cost. However, this requires a long development time. Rapid-Control-Prototyping (RCP) is a complete solution to develop, control, optimize, and test control strategies quickly and efficiently. Also, this technique provides a straightforward identification tool for research and development in the power electronics industry [28-33]. To provide an RCP for Real-Time Simulation (RTS), sophisticated control platforms are used in the research and industrial application development, such as dSPACE controllers, OPAL-RT, RT-Box controller, and Imperix. Despite their prices remaining high, those platforms' main advantages are based on a fast, simple, and efficient environment thanks to high-level programming. dSPACE remains the most popular on the market for its ease of use and integrates a software and hardware environment more suited to models designed with MATLAB/Simulink.

In this context, an RCP of an SPV water pumping system is proposed to save time and effort in implementing the proposed solution. It is reposed on the use of a DS1104 card from dSpace. The design of an overall circuit is composed of a DC-DC boost converter, a three-phase inverter, and an IM coupled to the centrifugal pump. From the control point of view, the system is based on a modified fractional open-circuit voltage (FOCV) MPPT method and V/f control to reduce the requirement of measurements. Only the PV voltage and the IM voltages are required. An experimental relationship based on temperature measurement is developed to avoid using the static switch for open-circuit voltage detection in the FOCV MPPT technique. Thus, ensures better power extracted at the output of the PV generator. The RCP technique is used to control, test, and validate the effectiveness of the complete system. Therefore, the proposed offgrid SPV water pumping system is built on a dSPACE DS1104 environment for a real-time command, visualization, and data acquisition. Experimental results verify the effectiveness of the proposed strategy. The SPV water pumping has been tested under different operating regimes exhibiting proper behavior.

The rest of the paper is organized as follows: the modelling of SPVWPS is presented in Section 2 that contains detailed designs and calculations. The proposed control strategy is explained in Section 3, while the experimental results are discussed in Section 4; the conclusions are drawn in Section 5.

\section{Modelling of SPVWPS}

In this fragment, the design and configuration of the SPVWPS are described in detail. It also demonstrated the mathematical models of all functional blocks related to this SVPWPS which can be realized for the experimental validation. 
2.1. System Design and Configuration. The proposed installation of high flow SVPWPS comprises SPV panels that act as a DC source, electronic conversion device, motor pump, and command and control system. The complete architecture of the proposed off-grid scheme is depicted in Figure 1. follows.

The expanded description of the functional blocks is as

(i) A PV generator was built based on ten panels connected in series. Their main specifications are given in Table 1

(ii) A SEMIKRON DC-AC inverter of rated power $20 \mathrm{KVA}$. This device is designed for both DC/DC converter and three-phase VSI. The control signals are generated using a dSPACE 1104 Digital Signal Processor "DSP" board. The PWM command of the boost converter is generated using the MPPT controller, where the inverter is controlled using the SVPWM technique

(iii) A dSPACE DS1104 is used as an RCP platform. The advantage of this controller is the relatively simple implementation of control strategies using the MATLAB/Simulink environment

(iv) A Lowara CEAM 70/3/A motor pump set consisting of a three-phase squirrel-cage motor connected in star and coupled to a single-impeller centrifugal pump. The scalar control, well known $V / f$ control, is proposed to deliver reference speed to the IM. The characteristics of the motor pump are given in Table 2

(v) A water tank is used to simulate a mechanical load

(vi) A reduced number of voltage and current sensors for verification are used. Temperature and irradiation sensors are used in the PV array

2.2. PV Source Model and Simulation. The electrical equivalent circuit of the PV cell is equivalent to a current source coupled with a diode, a shunt resistor, and a series of resistance, as presented by Figure 2 [34].

The current $I_{\mathrm{ph}}$ is the current emitted by the cell under solar radiation; it is the current of photons. It is strongly related to the intensity of the insolation and depends only very slightly on the temperature variation. The current $I_{d}$ the diode is strongly related to the temperature and the energy of the gap of the junction. It also depends on the voltage of the diode. The shunt current $I_{\mathrm{sh}}$ corresponds to the current passing in the parallel resistor $R_{\mathrm{sh}}$.

The mathematical model describing the IV characteristics of a PV cell is given in Equation (1) $[16,21,22,34]$.

$$
I_{\mathrm{pv}}=I_{\mathrm{ph}}-I_{s}\left[\exp \frac{q\left(V_{\mathrm{pv}}+R_{s} I_{\mathrm{pv}}\right)}{\mathrm{NKT}}-1\right]-\frac{V_{\mathrm{pv}}+R_{s} I_{\mathrm{pv}}}{R_{\mathrm{sh}}}
$$

where $I_{\mathrm{pv}}, I_{\mathrm{ph}}$, and $I_{s}$ represent the PV array currents, the photocurrent, and the diode reverse saturation current, respectively. $q$ is the electron charge $\left(1.6021710^{-19} \mathrm{C}\right), V_{\mathrm{pv}}$ is the global voltage of PV array, $K$ is the Boltzmann constant equal to $1.380650310^{-23} \mathrm{j} / \mathrm{k}, T$ is the temperature of a $\mathrm{P}-\mathrm{N}$ junction in Kelvin, $N$ is the diode ideality constant, and $R_{s}$ and $R_{\mathrm{sh}}$ are the series and shunt resistors of the cell, respectively.

The PV panel characteristics are shown in Figure 3 that exhibit nonlinear behaviors related to temperature and insolation conditions. These characteristics are loaded with a resistor under standard test conditions (STC). This figure proves that there is only one operating point that corresponds to the maximum power, called maximum power point "MPP".

2.3. DC/DC Boost Converter Model and Simulation. A boost chopper is used to optimize the power supplied by the PV generator. Figure 4 illustrates a DC/DC boost converter $[35,36]$. The capacitor is modelled by its capacitance $C$ and series resistance $R_{C}$ while the inductor is modeled by its inductance $L$ and series resistance $R_{L} \cdot R_{C}$ and $R_{L}$ are assumed insignificant in this design. Likewise, let us admit the transistor ideal and the effect of the forward voltage across the diode is neglected during conduction (where $R$ is the load resistor).

For the last proposed scheme of a DC/DC boost converter, a dynamic system can be described by the following equations:

$$
\left\{\begin{array}{l}
\frac{d v_{O}}{d t}=\frac{(1-D)}{C} i_{L}-\frac{1}{R C} v_{O}, \\
\frac{d i_{L}}{d t}=\frac{1}{L} v_{\text {in }}-\frac{(1-D)}{L} v_{O} .
\end{array}\right.
$$

The steady-state regime assumes the following:

(i) The average current in the capacitor is equal to zero, and therefore, the average current in the load is equal to the average current in the diode

(ii) The average voltage across the inductor is reduced to the voltage drop in its resistance; it is generally considered null

(iii) The average voltage across the capacitor is equal to the voltage of the load

(iv) The average voltage across the diode is equal to the difference between the input voltage and the load

$$
\left\{\begin{array}{l}
I_{C}=0=I_{D}-I_{O}=I_{D}-\frac{V_{O}}{R}, \\
V_{L}=R_{L} I_{L} \simeq 0, \\
V_{C}=V_{O}=R I_{O}=R I_{D}, \\
V_{D}=V_{\text {in }}-V_{L}-V_{O}=V_{\text {in }}-V_{O} .
\end{array}\right.
$$

Let us also note that there are two modes of waveforms of the inductance current. These modes are, respectively, called discontinuous conduction mode (DCM) and 


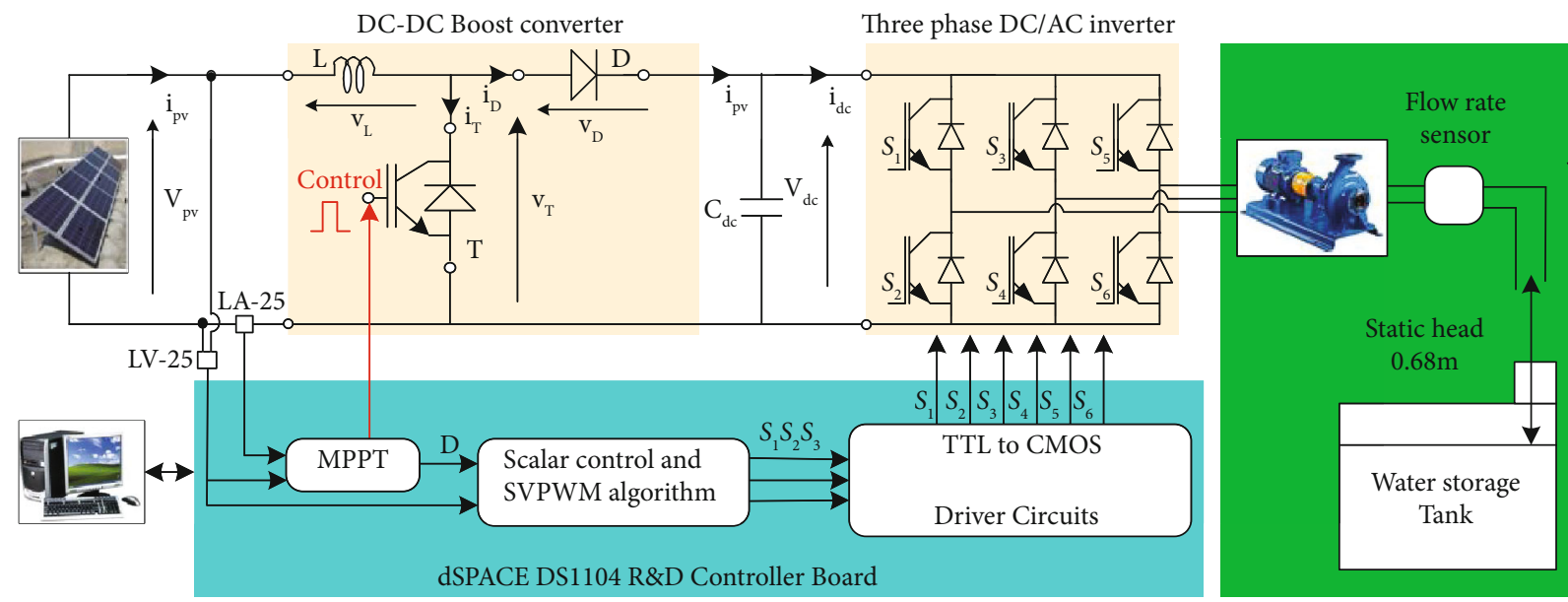

FIGURE 1: The system architecture of the proposed off-grid SPV water pumping system.

TABLE 1: PV module specifications.

\begin{tabular}{lc}
\hline Characteristic & Value \\
\hline Maximum power $\left(P_{\mathrm{MPP}}\right)$ & $50 \mathrm{Wp}$ \\
Optimal voltage $\left(V_{\mathrm{MPP}}\right)$ & $17.2 \mathrm{~V}$ \\
Optimal current $\left(I_{\mathrm{MPP}}\right)$ & $2.9 \mathrm{~A}$ \\
The voltage at the open circuit $V_{\mathrm{OC}}$ & $20 \mathrm{~V}$ \\
Photocurrent $\left(I_{\mathrm{SC}}\right)$ & $3.4 \mathrm{~A}$ \\
\hline
\end{tabular}

TABLE 2: Motor-pump characteristics.

\begin{tabular}{lc}
\hline Characteristic & Value \\
\hline Pump power rating & $0.37 \mathrm{KW}$ \\
IM power rating & $0.61 \mathrm{KW}$ \\
Voltage rating & $220 / 380 \mathrm{~V}$ \\
Current rating & $2.6 \mathrm{~A}$ \\
Speed rating & $2800 \mathrm{rpm}$ \\
\hline
\end{tabular}

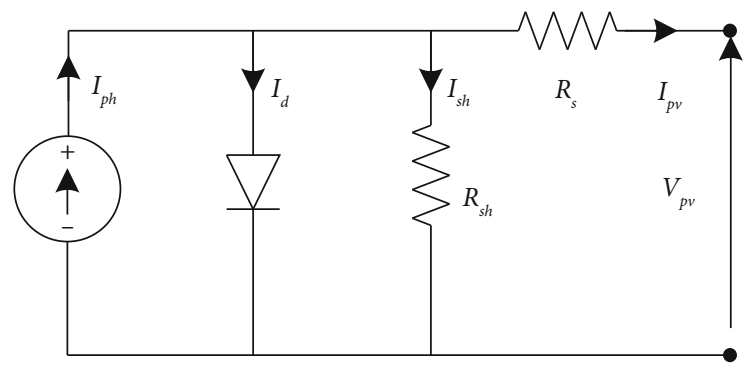

Figure 2: Solar cell equivalent circuit.

continuous conduction mode (CCM). In the case of DCM, the current $i_{L}(t)$ is cancelled before the cancellation of the commutation period. On the contrary, in the case of CCM, the minimum value of $i_{L}(t)$ is more significant or at the limit equal to zero. In this work, we assume that our boost converter is operating in the CCM regime. The output voltage of the converter is then governed as follows:

$$
V_{O}=\frac{1}{1-D} V_{\text {in }}
$$

The value of the inductor $L_{\min }$ selected based on estimated inductor ripple current $\Delta I_{I_{L}}$ at a maximum input voltage $V_{\text {in }}$ is given in Equation (5), with quantity $f_{s}$ designates the switching frequency.

$$
L_{\min }=\frac{D}{f_{s} \Delta I_{I_{L}}} V_{\text {in }} .
$$

The minimum value of capacitance $C_{\min }$ that results in the output voltage ripple $\Delta V_{O}$ is given by:

$$
C_{\min }=\frac{D}{f_{s} R \Delta V_{o}} V_{O} \text {. }
$$

The optimum load corresponding to the $100 \%$ efficiency of the converter is expressed as follows:

$$
R_{\mathrm{opt}}=\frac{1}{(1-D)^{2}} \frac{V_{\text {in }}}{I_{L}} .
$$

Figure 5 provides the waveforms of the inductance current, the current of the diode, and the inductance voltage, respectively.

2.4. DC-Link Voltage Calculation/DC-Bus Voltage Calculation. For a three-phase voltage inverter whose output voltage $V_{s}$ can reach $230 \mathrm{~V}$ RMS value, the DC bus voltage $V_{\mathrm{dc}}$ must obey the following relationship:

$$
V_{\mathrm{dc}}=\sqrt{2} V_{s}=\sqrt{2} \times 330=325 \mathrm{~V} \text {. }
$$

The DC bus voltage must be greater than $325 \mathrm{~V}$; therefore, a voltage $V_{\mathrm{dc}}$ of $400 \mathrm{~V}$ can operate the inverter in the best conditions. 


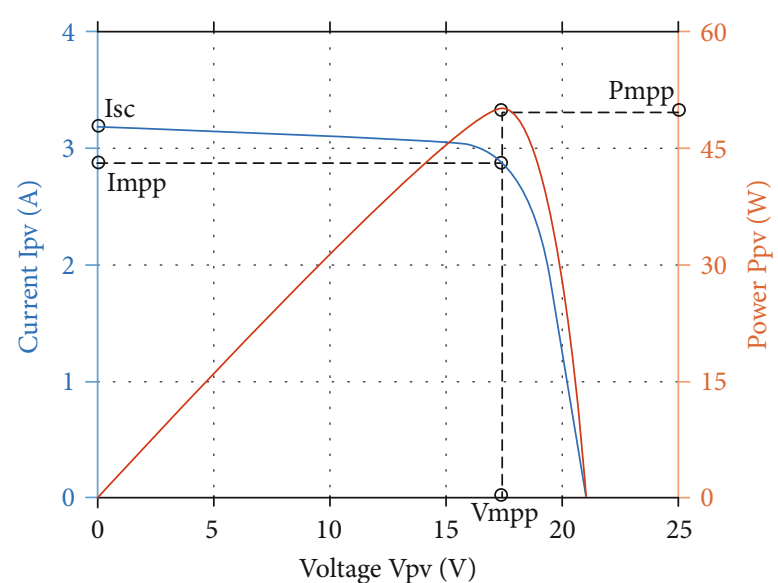

FIGURE 3: STC characteristics.

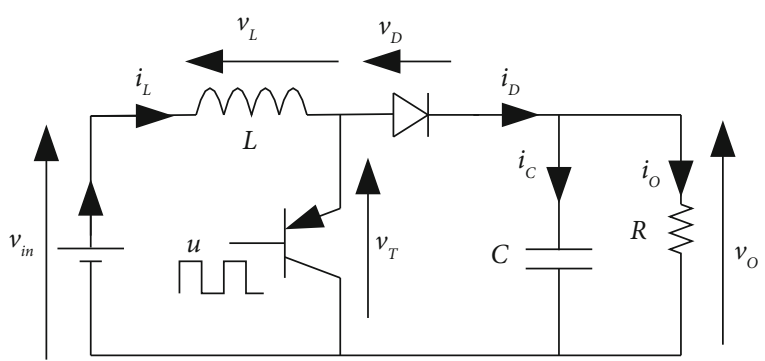

FIgURE 4: Circuit diagram of DC/DC boost converter.
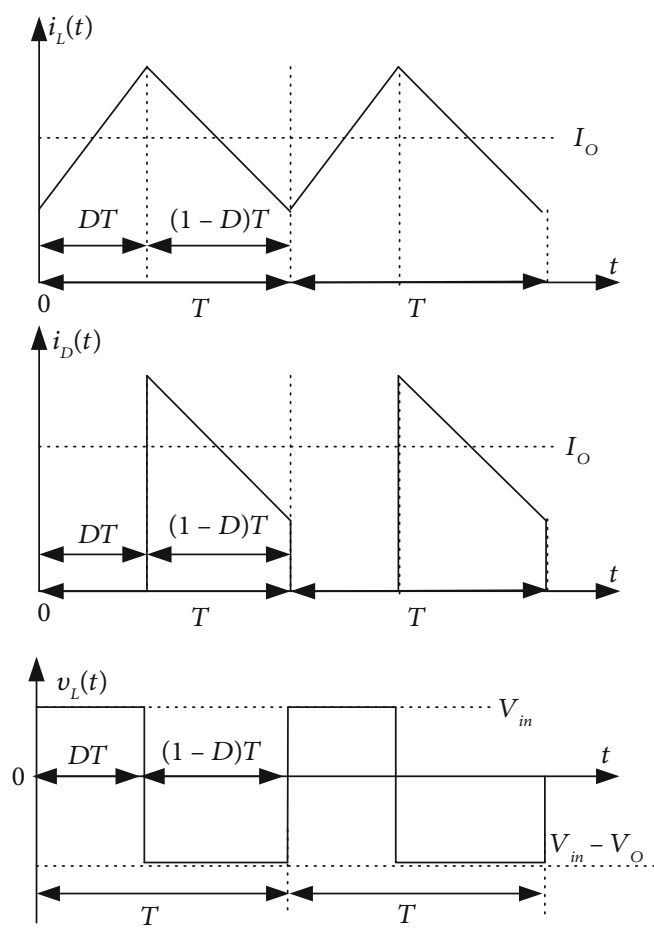

Figure 5: Boost converter waveforms at CCM.
2.5. Design of Boost Converter Parameters. The duty ratio ( $D$ ) for the boost converter is calculated as follows [37]:

$$
D=\frac{V_{O}-V_{\text {in }}}{V_{O}}=1-\frac{325}{400}=0.38
$$

Let us assume that the boost converter operates at the MPP and STC conditions; $V_{\mathrm{pv}}=V_{\mathrm{MPP}}, I_{\mathrm{pv}}=I_{\mathrm{MPP}}$, the optimum load resistor $R_{\mathrm{opt}}$ is

$$
R_{\mathrm{opt}}=\frac{1}{(1-D)^{2}} \frac{V_{\mathrm{MPP}}}{I_{\mathrm{MPP}}}=\frac{1}{(1-0.38)^{2}} \frac{172}{2.9}=150 \Omega \text {. }
$$

For a current ripple $\Delta I_{I_{L}}$ designed of $10 \%$, the minimum value of the inductor is

$$
L_{\text {min }}=\frac{D}{f_{s} \Delta I_{I_{L}}} V_{\mathrm{MPP}}=\frac{0.38}{10000(2.9 \times 0.1)} 172=22.5 \mathrm{mH} .
$$

The minimum value of the capacitance corresponds to $1 \%$ of the voltage ripple $\Delta V_{O}$ and load resistor with $150 \Omega$ is

$$
C_{\text {min }}=\frac{D}{f_{s} R_{\mathrm{opt}} \Delta V_{o}} V_{O}=\frac{0.38 \times 325}{10000 \times 150 \times(325 \times 0.01)}=25.33 \mu \mathrm{F} .
$$

Hence, a capacitance of $50 \mu \mathrm{F}$ is sufficient. In summary, an inductor of $22.5 \mathrm{mH}$ and a capacitance of $50 \mu \mathrm{F}$ can be chosen.

2.6. Design of DC-Link Capacitor. The treated system is designed for water pumping; therefore, the DC-link capacitor $C_{\mathrm{dc}}$ connected between the boost converter and the VSI is estimated according to the fundamental frequency as follows [37]:

$$
\begin{gathered}
\omega_{\text {rated }}=2 \pi f_{\text {rated }}=100 \pi=314 \mathrm{rad} / \mathrm{s}, \\
C_{\mathrm{dc}}=\frac{6 a V I t}{\left(V_{\mathrm{dc}}^{2}-V_{\mathrm{dcl}}^{2}\right)}=\frac{6 \times 1.2 \times 132.8 \times 2.9 \times 0.005}{\left(400^{2}-325^{2}\right)}=254.976 \mu \mathrm{F} .
\end{gathered}
$$

Therefore, a capacitor of $250 \mu \mathrm{F}$ and $400 \mathrm{~V}$ is selected. In the previous relationship, $V_{\mathrm{dc}}$ is the estimated dc voltage, $V_{\mathrm{dcl}}$ is the selected dc voltage during transients, $a$ is the overloading factor, and $t$ refers to the time by which selected voltage reach at estimated voltage range. Furthermore, $V$ and $I$ designate the phase voltage and current of the motor, respectively.

2.7. Three-Phase DC/AC Inverter Model. The IM is driven by a three-phase VSI $[13,33,37]$, whose objective is to furnish in the output a variable voltage and frequency through a PWM controller $\left(S_{1}-S_{6}\right.$ is the power switch). The switches of any leg of the inverter are complementary. Various PWM techniques can be exploited to produce control signals for a voltage inverter. This work uses the space vector PWM 
technique. The SVPWM technique is commonly used for the application of scalar speed control methods. The comparison with other PWM, SVPWM offers better performance because it offers less total harmonic distortion (THD), lower switching losses at the high switching frequency, and enables efficient use of DC-link voltage [4, $11,18]$.

The principle of PWM is explained by using the VSI block diagram shown in Figure 1. For a three-phase VSI admitted without loss, the output voltage is strictly defined by the voltage of the DC bus $V_{\mathrm{dc}}$ supplying the inverter and by the logic state of the three highest IGBT's $\left(S_{1}, S_{2}, S_{3}\right.$ ) . With the three Boolean variables $S_{1}, S_{2}$, and $S_{3}$, there are only eight possible combinations. From these combinations, we determine eight voltage vectors, six active vectors denoted from $\bar{v}_{1}$ to $\bar{v}_{6}$, and two zero vectors $\bar{v}_{0}$ and $\bar{v}_{7}$. It is easily observed that the active vectors form a balanced six-phase system; therefore, the same modulus and chronic phases equal to $60^{\circ}$. Indeed, it is possible to pose the following system of equations where $k$ is an integer indicating the switching combinations.

$$
v_{k}= \begin{cases}\sqrt{\frac{2}{3}} V_{\mathrm{dc}} e^{j(k-1) \pi / 3} & \text { for } k=1, \cdots, 6, \\ 0 & \text { for } k=0,7 .\end{cases}
$$

Figure 6 below shows the general principle of the SVPWM technique. Figure 6(a) gives a spatial representation of these vectors while indicating each vector's associated combination of commands. For example, the combination 010 corresponds to $S_{1}=0, S_{2}=1$, and $S_{3}=0$. The SVPWM technique exploits the vector voltages diagram and synthesizes a requested reference voltage vector $\bar{v}_{\text {ref }}$ from the two neighboring vectors $\bar{v}_{k}$ and $\bar{v}_{k+1}$ and the null vectors $\bar{v}_{0}$ or $\bar{v}_{7}$ as indicated in Figure 6(b). This synthesis must be done in average value over a time interval $T_{s}$. The vectors $\bar{v}_{k}$ and $\bar{v}_{k+1}$ are applied during the time intervals $\tau_{k}$ and $\tau_{k+1}$, respectively, and the null vector is applied during the remaining time $\tau_{0}$, where $\tau_{0}=T_{s}-\tau_{k}-\tau_{k+1}$.

In other words, for an average value equivalent to $\bar{v}_{\text {ref }}$ over the period $T_{s}$, the following relation must be verified.

$$
\frac{\tau_{k} \cdot \bar{v}_{k}+\tau_{k+1} \cdot \bar{v}_{k+1}}{T_{s}}=\bar{v}_{\text {ref }} .
$$

To respect the constraint $\tau_{k}+\tau_{k+1} \leq T_{s}$, the $V_{\text {ref }}$ the module of the required voltage $\bar{v}_{\text {ref }}$ must verify the equation below.

$$
V_{\text {ref }} \leq \frac{V_{\mathrm{dc}}}{\sqrt{2}}
$$

In this context, the synthesis solution is given in Equa- tion (17), where the coefficient $\rho$ nominates a voltage ratio.

$$
\left\{\begin{array}{l}
\tau_{k}=T_{s} \rho \sin \left(\frac{\pi}{3}-\varsigma\right), \\
\tau_{k+1}=T_{s} \rho \sin (\varsigma), \\
\rho=\frac{\sqrt{2} V_{\mathrm{ref}}}{V_{\mathrm{dc}}}
\end{array}\right.
$$

2.8. Association Motor-Pump Model. A squirrel-cage IM is used in this application. In a stationary reference frame, it is described by five main equations as follows [38]:

$$
\begin{gathered}
\frac{d \bar{\phi}_{s}}{d t}=\bar{v}_{s}-R_{s} \bar{i}_{s}, \\
\frac{d \bar{\phi}_{r}}{d t}=j \omega_{r} \bar{\phi}_{r}-R_{r} \bar{i}_{r}, \\
\bar{\phi}_{s}=\ell_{s} \bar{i}_{s}+m \bar{\phi}_{r}, \\
T_{e}=-p \Im_{m}\left(\bar{\phi}_{s} \bar{i}_{s}^{*}\right)=p\left(\phi_{d s} i_{q s}-\phi_{q s} i_{d s}\right), \\
T_{e}-K_{f} \omega_{r}-T_{r}=\frac{j}{p} \frac{d \omega_{r}}{d t} .
\end{gathered}
$$

In the collection of equations above, variables and parameters with index " $s$ " are at the stator while those with " $r$ " are at the rotor. $\bar{v}, \bar{i}$, and $\bar{\varphi}$ designate the vectors of voltage, current, and flux, respectively. $R$ is the resistance, $\ell_{s}$ is the leakage inductance, and $m$ denotes the ratio $M / L_{r}$, where $M$ is the mutual inductance and $L_{r}$ is the rotor inductance.

Electrical speed and pole pair number are $\omega_{r}$ and $p . T_{e}$ and $T_{r}$ are electromagnetic and mechanical torques, respectively. Various equations can determine the electromagnetic torque, the best known of which is that of Equation (21) where $\mathfrak{\Im}_{m}$ designates the imaginary part; $\varphi_{d s}, \varphi_{q s}, i_{d s}$, and $i_{q s}$ are the direct and quadrature components of stator flux and current, respectively. This equation is common for transient stationary and steady-state $[14,38]$. The IM mechanical equation is expressed by Equation (22). Here, $K_{f}$ is the viscous coefficient, and $j$ is the moment of inertia $\left(\mathrm{kg} \mathrm{m}^{2}\right)$. The centrifugal pump driven by the IM develops a mechanical torque whose dynamics are presented by the following equation [12]:

$$
T_{r}=J \frac{d \omega_{r}}{d t}+K_{f} \omega_{r}+K \omega_{r}^{2} .
$$

Let it be noted that the steady-state torque produced by the IM is equivalent to the load torque of the pump $T_{r}$, the rotor speed is $\omega_{r}$ in $\mathrm{rad} / \mathrm{s}$, and $K$ is the centrifugal pump constant. For the water pump used in this test, the proportionality constant $K$ is given according to Equation (24).

$$
K=\frac{T_{r}}{\omega_{r}^{2}} .
$$

For the IM, the rated torque is $1.2619 \mathrm{Nm}$, and the rated 


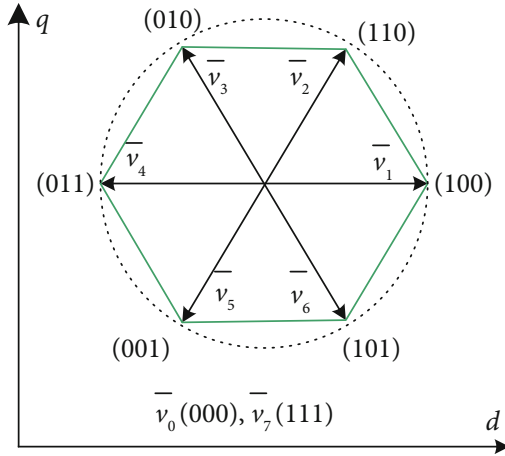

(a)

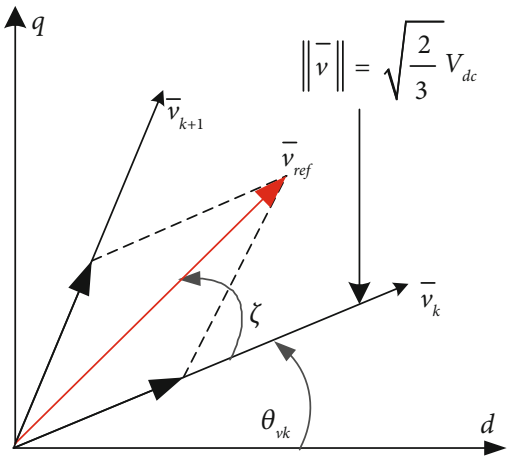

(b)

FIGURE 6: Principle of the SVPWM technique: (a) the voltage space vectors of the three-phase inverter; (b) synthesis of the SVPWM.

speed is $2800 \mathrm{rpm}$; thus, the proportionality constant $K$ evaluated based on (24) is equal to $14.67710^{-6} \mathrm{Nm} /(\mathrm{rad} / \mathrm{s})^{2}$. A KOBOLD C34P flow meter installed on the pump is used to measure the flow rate $(Q)$. The sensor is equipped with a display that delivers a flow rate varying from 0 to $100 \mathrm{l} / \mathrm{min}$. As an indication, a tank with $0.68 \mathrm{~m}$ of the head was used in the test bench as a well. The parameters are identified by the variation of the motor supply voltage, which drives the pump at a fixed frequency. However, the IM is connected to the grid via an autotransformer, i.e., a variable supply voltage and a fixed frequency of $50 \mathrm{~Hz}$. Table 3 illustrates the results obtained during the experiment.

The pumping performance of a centrifugal pump is expressed in the form of an $(H-Q)$ curve, depicting the flow $Q$ (e.g., in $\mathrm{m}^{3} / \mathrm{h}$ ) and the head $H$ (e.g., in $\mathrm{m}$ ) of the pump. The variation of the pump's speed can give us numerous characteristics $(H-Q)$ as described by the following relation $[25,39]$ :

$$
H=B_{1} \omega_{r}^{2}-B_{2} \omega_{r} Q-B_{3} Q^{2} .
$$

The optimal values of $B_{1}, B_{2}$, and $A B_{3}$ seek for $H=0.68$ $\mathrm{m}$ is $B_{1}=0.039, B_{2}=-0.3079$, and $B_{3}=-0.0024$.

Indeed, the results are used to plot the flow characteristic as a function of speed, Figure 7, and using the "polyfit" function of MATLAB library, the coefficients $a_{0}, a_{1}, a_{2}$, and $a_{3}$ of the equivalent polynomial of flow rate-speed relationship corresponding to Equation (26) are calculated.

$$
\begin{gathered}
Q=a_{3} N^{3}+a_{2} N^{2}+a_{1} N+a_{0}, \\
a_{3}=1.7 \times 10^{-9}, \\
a_{2}=9.3 \times 10^{-7}, \\
a_{1}=24 \times 10^{-3}, \\
a_{0}=-64 \times 10^{-3} .
\end{gathered}
$$

A first impression to exploit these results would be to control the flow to the speed. Since the behavior of the system is not linear, it would be interesting to program a speed control routine to ensure equivalent flow control.
2.9. Proposed Control Strategy. The model developed above of the SPVWPS needs a better control scheme to optimize its performance. The proposed strategy embodies the MPPT method for maximum power extraction from the PV generator by the real-time control of the duty ratio of a boost converter, a constant $V / f$ control to give the reference speed of the IM drive, and an SVPWM algorithm to control the power semiconductor devices for better use of dc-bus voltage and a minimum THD.

2.10. Proposed MPPT Structure. The MPPT structure proposed and carried out in this work is the FOCV method. This choice is based on several factors, such as this method is of a simple structure. Low cost, remarkably achievable analogically, and the ability of its easy integration with a DC/DC controller in various applications like the SPVWPS. FOCV method [16, 17, 19, 21, 25-27], based on the use of reference voltage to adjust the duty ratio of the MPPT controller in a feedback control loop, supposes that irradiation and temperature variations on the array have not a significant impact on the MPP voltage value and that MPP voltage can be estimated with a predefined constant voltage $V_{\mathrm{REF}}$. Thus, the optimum voltage is always close to a proportion of the open circuit voltage $V_{\mathrm{OC}}$. Consequently, $V_{\mathrm{REF}}$ can be calculated from the empirical relationship shown in Equation (31). It is found that the value of $K$ varies between $72 \%$ and $78 \%[17,26]$.

$$
V_{\mathrm{REF}}=K V_{\mathrm{OC}} .
$$

In this method, the error arising from the comparison of the array voltage with a constant reference voltage is used to continuously adjust the converter duty cycle so that the array operates at a predetermined operating point near the MPP, as seen in Figure 8.

This figure shows that after $V_{\mathrm{OC}}$ is sampled by a sampler; $V_{\mathrm{REF}}$ which is calculated using Equation (31) is kept constant during one sampling period by hold circuit; now duty ratio $D$ is adjusted to make $V_{\mathrm{pv}}=V_{\mathrm{REF}}$. For the following sample, again $V_{\mathrm{OC}}$, it is sampled, and the same procedure is repeated for each sample.

The implementation of the FOCV technique needs the measurement of open-circuit voltage $V_{\mathrm{OC}}$ for all climatic 
TABLE 3: Measurement of flow under supply voltage variation.

\begin{tabular}{lcc}
\hline Voltage $(\mathrm{V})$ & Speed $N(\mathrm{tr} / \mathrm{min})$ & Flow rate $Q(\mathrm{l} / \mathrm{min})$ \\
\hline 118 & 1242 & 27 \\
136 & 1482 & 33.2 \\
180 & 1904 & 38.5 \\
230 & 2167 & 38.7 \\
280 & 2310 & 39.3 \\
330 & 2385 & 38.2 \\
380 & 2738 & 38 \\
\hline
\end{tabular}

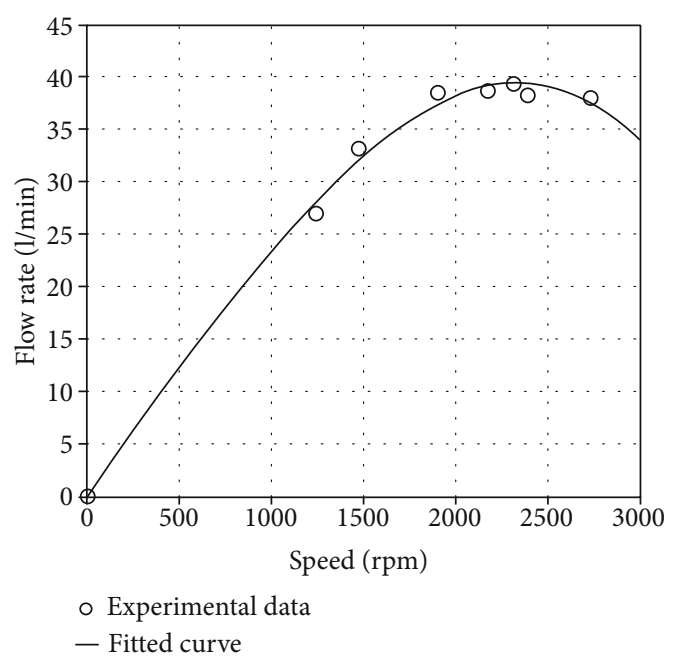

FiguRE 7: Identification of the flow-speed characteristic.

change. Therefore, it is mandatory to use a static switch in series with the PV generator to establish the open-circuit state. This produces a large oscillation of the output power of the PV array.

Because the voltage $V_{\mathrm{OC}}$ varies essentially with temperature $\left(V_{\mathrm{OC}}\right.$ decreases in a slight way, unlike the increase of the temperature), this drawback can be eliminated by measuring only the temperature according to giving relation. To overcome this inconvenience, a series of measurements is carried out on the variation of the $V_{\mathrm{OC}}$ and the optimal voltage $V_{\text {MPP }}$ as a function of temperature, see Table 4 .

Figure 9 confirms that $V_{\mathrm{OC}}$ decreases slightly when the temperature increasing and can be approximated by the following linear function:

$$
V_{\mathrm{OC}}=a T+b,
$$

where $a=-0.1754$ and $b=23.6443$.

On the other hand, Table 4 shows that the constant $K$ of Equation (31) is 0.77 , and the average value of the optimal voltage is around $14.72 \mathrm{~V}$ in this practical study.

The flowchart of the modified FOCV method is presented in Figure 10. In this figure, the temperature is measured in parallel with the measurement of the voltage of the PV generator to calculate the voltage $V_{\mathrm{OC}}$ then the reference voltage. Thus, the maximum power is tracked by com- paring the current PV voltage to the already calculated reference voltage.

2.11. Scalar (V/f) Control for IM. The scalar control of an IM is the most prevalent and easiest to date. This approach, also called $V / f$ control, is easy to implement and costeffective. With the exception of $V / f$ control, FOC and DTC algorithms are difficult and involve additional current sensors for their implementation $[9,13,38,40,41]$. The $V /$ $f$ control focuses only on the steady-state model of the IM and assumes that the magnitude of stator flux remains constant in this operating mode. The speed regulation of the motor can be achieved by adjusting the stator voltage magnitude and frequency in such a way that the air gap flux is always conserved at the requisite value. According to Equation (18) at a steady-state and within the low frequency, the voltage drop at the stator resistance can be neglected. Therefore, by keeping the ratio between stator voltage $V_{s}$ and stator field frequency $f_{s}$ constant, the magnitude of stator flux $\Phi_{s}$ is controlled as shown in Equation (33) [37, 41], where $K_{v}$ is the constant of proportionality between the nominal voltage $V_{n}$ of phase and frequency $f_{n}$ of the voltage source.

$$
\Phi_{s} \approx \frac{V_{s}}{2 \Pi f_{s}}=K_{v} .
$$

At low operating frequency under a few Hertz, the voltage drop across to the stator resistance is no longer negligible compared to the leakage reactance. This structure of control should consider the voltage drops to keep a constant stator flux. Inversely, to protect the motor against overvoltage if the rotor speed is more significant than that which corresponds to the rated frequency $f_{n}$, the stator voltage must be adjusted accordingly according to the following relation:

$$
V_{s}= \begin{cases}\left(V_{n}-V_{0}\right) \frac{f_{s}}{f_{n}}+V_{0} & \text { for } f_{s}<f_{n}, \\ V_{n} & \text { for } f_{s} \geq f_{n} .\end{cases}
$$

Here, $V_{0}$ denotes the stator voltage at zero frequency. The presented system tracks the MPP by the FOCV MPPT structure proposed and described above so that the motopump can extract the maximum power available from the SPV array for all solar insolation changes. The MPP tracker is designed here based on the boost converter. So, the output voltage average value of the DC-DC boost converter $V_{\mathrm{dc}}$ in CCM mode is expressed in terms of the average value of the input voltage $V_{\mathrm{pv}}$ by Equation (4). The following equation gives the voltage magnitude of the IM. Here, $\rho$ denotes the voltage ratio and $D$ the duty ratio adjusted by the controller of the MPP.

$$
V_{s}=\rho V_{\mathrm{dc}}=\frac{\rho}{1-D} V_{\mathrm{pv}}=\delta V_{\mathrm{pv}} .
$$

Thus, the stator reference pulsation $\omega_{\text {sref }}$ is obtained based on the $V_{s} / f_{s}$ technique. The resulting references of stator pulsation $\omega_{\text {sref }}$ and the voltage ratio $\rho$ are applied to the 


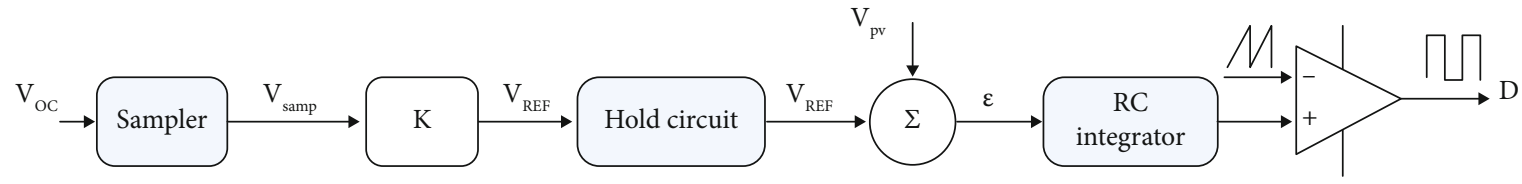

FIGURE 8: MPPT using the constant voltage method.

TABLE 4: $V_{\mathrm{OC}}$ and $V_{\mathrm{MPP}}$ voltage responses versus temperature.

\begin{tabular}{lccc}
\hline$T\left({ }^{\circ} \mathrm{C}\right)$ & $V_{\mathrm{OC}}(\mathrm{V})$ & $V_{\mathrm{MPP}}(\mathrm{V})$ & $K$ \\
\hline 22 & 19.86 & 15.344 & 0.7726 \\
23 & 19.72 & 15.202 & 0.7709 \\
26 & 18.92 & 14.584 & 0.7708 \\
28 & 18.54 & 14.260 & 0.7691 \\
32 & 18.2 & 14.201 & 0.7802 \\
\hline
\end{tabular}

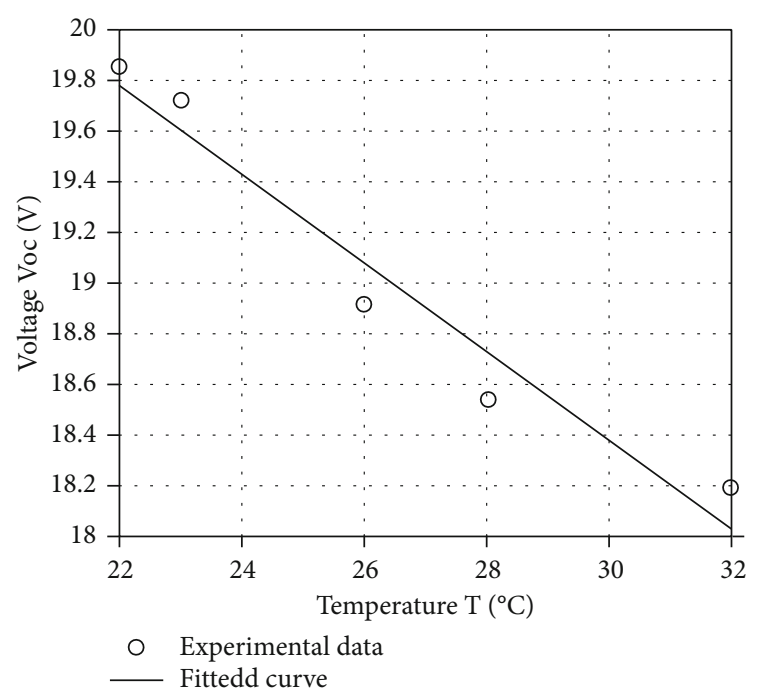

FigURE 9: $V_{\text {OC }}$ response versus temperature variation.

motor using an SVPWM technique driving three-phase twolevel VSI. The schematic diagram of the proposed control is exposed in Figure 11.

\section{Experimental Results and Discussions}

To check the functionality and analyse the performance of the designed system, an experimental investigation is performed for different insolation conditions. The whole algorithm is designed using the MATLAB/Simulink model and implemented in the dSPACE DS1104 environment. It comprises both the algorithms, likely MPPT algorithm for the power maximisation and the $V / f$ control along with the SVPWM algorithm. The prototype hardware setup was developed in the laboratory for experimental validation (Figure 12) using various elements such as DC-AC inverter, dSPACE DS1104, TTL-CMOS modules, sensors, a series connection 10 panels, motor-pump with a flow meter, and a tank.
The main program of the proposed application based on an RCP system is modelled using Simulink/MATLAB and implemented on the DS1104 R\&D Controller Board, as shown in Figure 13. The measured temperature is converted into a reference voltage and compared to the actual voltage of the solar panel to generate an error $\Delta V$. The sum of this error and its integral constitutes the PI regulator. The output of the PI regulator is compared to a triangular signal to give the command to the chopper via a galvanic isolation circuit. A vital building SVPWM block allows determining the time duration and the sector for each reference vector. The inputs of the SVPWM are generated based on the constant $V / f$ control principle. The stator voltage applied to the stator windings of the IM is determined according to the reference vector generated and the actual voltage of the DC bus $V_{\mathrm{dc}}$.

Indeed, other Simulink blocks are used for the acquisition of verification and validation data (insolation, flow rate, electromagnetic torque, currents, power, efficiency, etc.) and are not shown in Figure 13.

\section{Test Results for MPPT Solution}

The effectiveness of the FOCV technique is based on the use of a voltage sensor (LEM LV-25) for the acquisition of the PV array voltage $V_{\mathrm{pv}}$. Moreover, the simplest form of operation is to maintain the PV generator at a constant voltage identical to the optimal voltage at STC furnished by the manufacturer (neglecting the effects of solar radiation and temperature changes on the MPP voltage). Equations (31) and (32) are applied to obtain the adequate reference voltage for the system. Further, the required information on the temperature is perceived using an electronic circuit (LM35) installed in the roof adjacent to PV panels. The sensed voltage of the PV and the temperature are applied to two ADC's of the DS1104. The computer system internally calculates the reference voltage and compares it to the measured value to generate an error, and it can be compared to a triangular wave. Further, a PWM signal is generated for an insulated gate bipolar transistor (IGBT) using a boost converter. Then, the output voltage of the boost converter is used to determine the stator voltage $V_{s}$ that needs to be applied to the IM according to Equation (35). The centrifugal pump coupled with IM is maintained under a nominal constant stator flux of $0.7 \mathrm{~Wb}$. Therefore, stator pulsation $\omega_{s}$ is obtained in real-time by dividing the stator voltage $V_{s}$ by 0.7 ; it is keeping the rule $V / f$ constant.

The practical results obtained on the three-phase pumping system with an improved FOCV MPPT algorithm for a temperature of $24^{\circ} \mathrm{C}, 15$ minutes, and under insolation variation are summarized in the figures below. Figure 14(a) shows the arbitrary scenario captured for the insolation 




FIgURE 10: Flowchart of the modified FOCV MPPT method.

(Ins), whereas Figure 14(b) denotes the evolution of the duty cycle $D$ for the boost converter to reach the MPP in realtime. The insolation ranges from 500 to $850 \mathrm{~W} / \mathrm{m}^{2}$ generating a variable $D$ of 20 to $35 \%$ that is instantly adjusted to extract the maximum power according to the improved FOCV MPPT technique proposed. The instantaneous evolution of the input power $\left(P_{\mathrm{pv}}\right)$ and the optimal power $\left(P_{\mathrm{pv}-\mathrm{opt}}\right.$ ) that can be delivered by the SPV generator are presented in Figure 14(c). It is observed that they are confused that confirms that the system extract the maximum power available, and the efficiency is optimal and reaches $100 \%$. In this test situation, the maximum insolation is $850 \mathrm{~W} / \mathrm{m}^{2}$ correspond to an optimal current of $2.48 \mathrm{~A}$ (verified according to STC delivered by the manufacturer, Table 1), and according to Equations (31) and (32) for a temperature of $24^{\circ} \mathrm{C}$, the average MPP voltage is $150 \mathrm{~V}$; then, the maximum available power for the PV generator is $372 \mathrm{~W}$. This verification calculation is confirmed by Figure 14(d) that presents the movement of MPP against the optimum voltage. Zoom on this figure shows a better movement of the MPP with a maximum power of $375 \mathrm{~W}$ (Figure 14(e)).

Figure 14(f) displays that the PV voltage $V_{\mathrm{pv}}$ is maintained constant around their optimum reference $P_{\mathrm{pv}-\mathrm{opt}}$ under insolation variation. This figure proves that the variation of insolation does not have a significant impact on the MPP voltage value and that the boost converter provides a variable output voltage $\left(V_{\mathrm{dc}}\right)$ According to the duty cycle to reach the MPP. Figures 14(b) and 14(f) prove that for a value of $D$ equivalent to $35 \%$ as calculated previously by Equation (9), the voltage delivered $V_{\mathrm{dc}}$ is $231 \mathrm{~V}$.

It is a crucial task to study the effectiveness of the proposed algorithm on the complete pumping system. Therefore, the characteristics of stator voltage, pulsation response, stator current, and flow rate are studied, and the observed results are presented. Figure $14(\mathrm{~g})$ proves that the



Figure 11: Schematic diagram of $V / f$ control with FOCV MPPT structure.

rule of $V / f$ constant is respected because the stator voltage $V_{s}$ and the pulsation $\omega_{s}$ vary proportionally with a constant confirmed of $0.7 \mathrm{~Wb}$. It is noted in Figure 14(h) that stator current $\left(I_{s}\right)$ of the IM varies proportionally versus the power extracted from the PV array. Figure 14(i) proves that the flow rate $(Q)$ varies in proportion to the available power, and its maximum value accelerates and decelerates depending on the extracted maximum power.

A second experiment is saved for the same temperature but for insolation that considered constant at about $800 \mathrm{~W} / \mathrm{m}^{2}$, over 15 minutes of recording (Figure 15). We observe, respectively, the scenario of insolation for these test conditions, the duty cycle of the converter, the PV voltages and the DC bus voltage, the available PV power and the extracted one, the movement of the MPP point, the stator voltage and pulsation and the current magnitude, and the flow rate.

It is easy to see that the duty cycle is kept constant at about 0.325 which raises the average voltage at the boost converter output to $240 \mathrm{~V}$ while keeping the PV voltage equal to an optimal reference voltage around $150 \mathrm{~V}$. The power extracted is close to the optimal power available, which results in a maximum power operation and consequently an efficiency that exceeds $99 \%$. The system then operates on the MPP point as shown in Figure 15(e). The stator quantities are kept constant, and the voltage is proportional to the pulsation with a slope of $0.7 \mathrm{~Wb}$ in steady-state operation. Figure 15(h) shows that the water flow rate remains constant at its maximum value for this constant insolation operating condition, and therefore, the motor supply voltage is well controlled.

While comparing the performance of the conventional FOCV method where the reference voltage $\left(V_{\mathrm{REF}}\right)$ is imposed whatever the variation of the weather conditions or precalculated by measuring the open-circuit voltage ( $\left.V_{\mathrm{OC}}\right)$, the proposed structure makes it possible to directly find the reference voltage, which results in a better power extraction and reduced oscillations. Indeed, a third experiment is recorded by imposing a reference voltage $V_{\mathrm{REF}}$ of $142 \mathrm{~V}$. The results of this test are shown in Figure 16. 




(a)

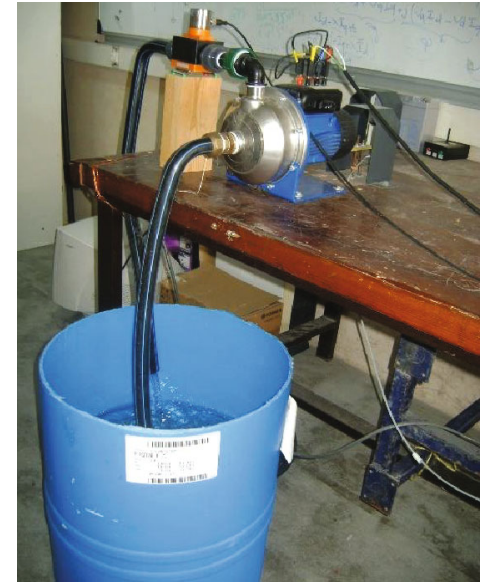

(b)

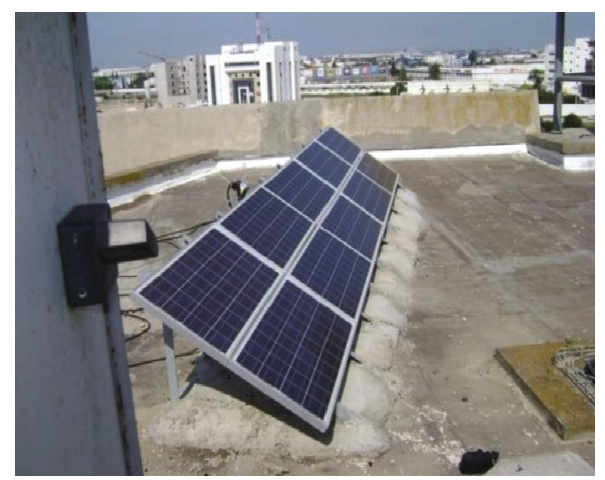

(c)

FIGURE 12: The hardware setup of the SPVWPS: (a) overview of the test bench; (b) motor pump with flow meter and water tank; (c) a series connection of 10 panels.

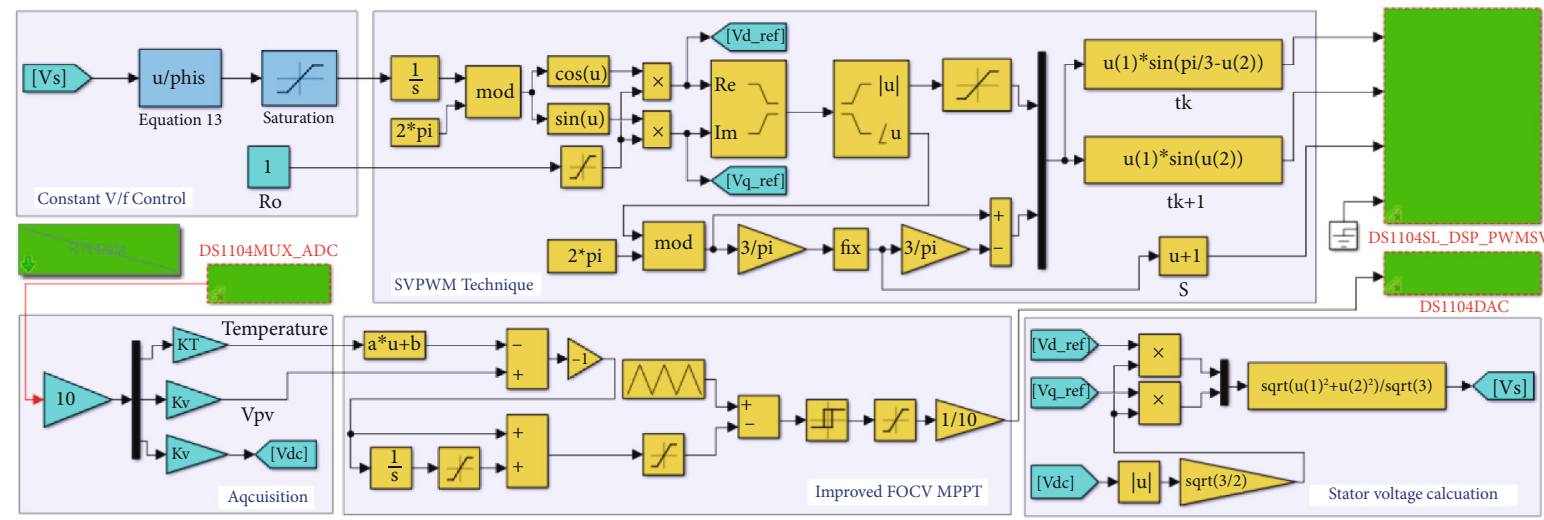

Figure 13: Simulink model of the implemented main RCP program.

Figure 16(a) shows that the efficiency of the boost converter $E_{f f}$ exceeds $91 \%$ and reaches $97 \%$ for a solar irradiance varying from 500 to $1000 \mathrm{~W} / \mathrm{m}^{2}$. This is confirmed by Figure 15(b) that presents the output power $P_{\mathrm{dc}}$ of the boost converter and the available one in the PV generator $P_{\mathrm{pv}}$. The reference voltage is kept constant around $142 \mathrm{~V}$ while the voltage at the output of the inverter $V_{\mathrm{dc}}$ varies according to the insolation to seek the MPP as shown in Figure 15(c). This objective is reached by the action on the duty cycle $D$. Figure 15 (d) shows that although the system can reach MPP, it shows large oscillations around the reference voltage set at $142 \mathrm{~V}$.

Based on the conventional FOCV MPPT technique, it is observed that the measured voltage varies from 134 to $150 \mathrm{~V}$ depending on the variation of the sunlight, leading to an oscillation of $11 \%$ compared to the imposed reference voltage. This causes heating of the power components and additional losses, and consequently, some power is lost. On the 


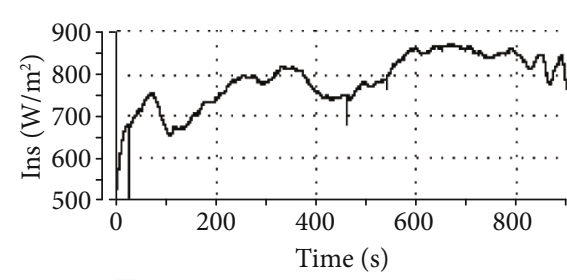

(a)

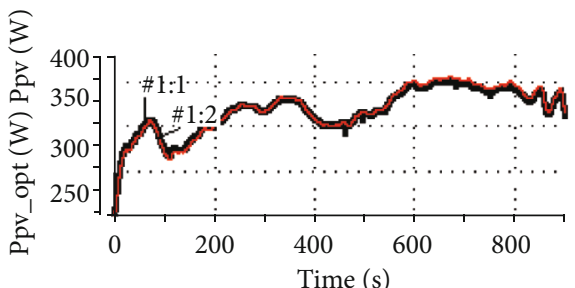

\#1:1 Ppv (Model Root/Ppv)

\#1:2 Ppv_opt (Model Root/Ppv_opt)

(c)

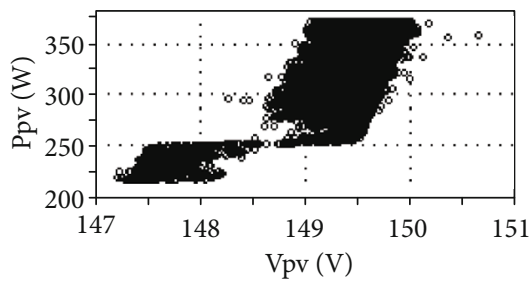

(e)

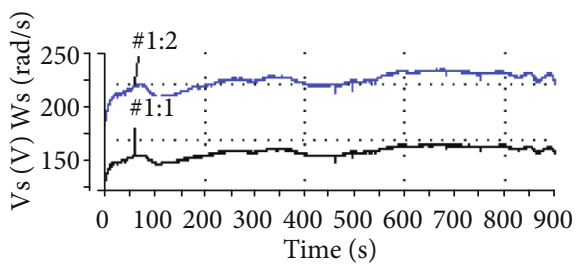

\#1:1 Vs (Labels/Vs)

\#1:2 Ws (Labels/Ws)

(g)

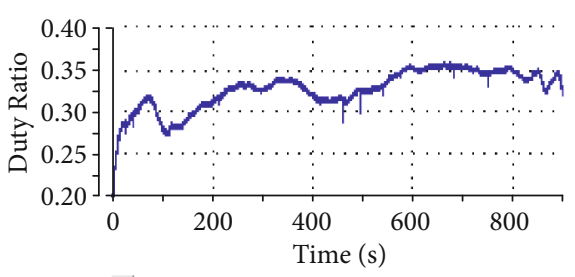

(b)

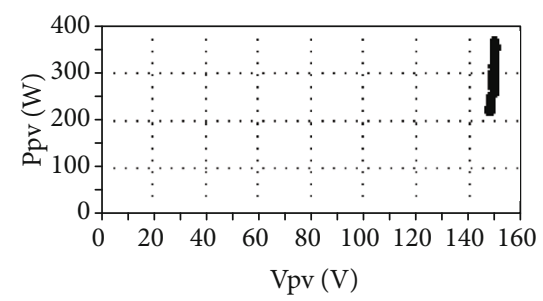

(d)

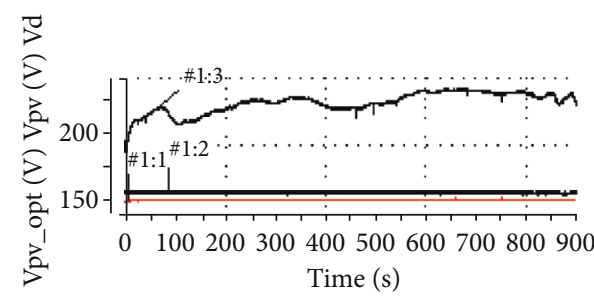

\#1:1 Vpv (Model Root/Vpv)

\#1:2 Vpv_opt (Model Root/Vpv_opt)

\#1:3 Vdc (Labels/Vdc)

(f)

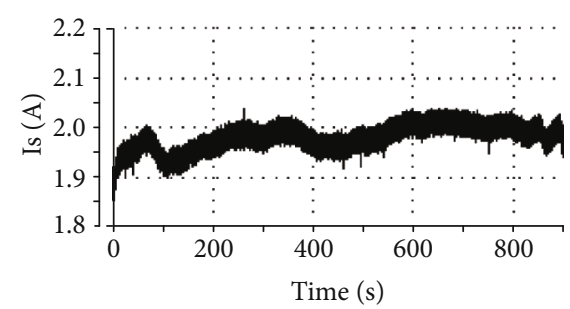

(h)

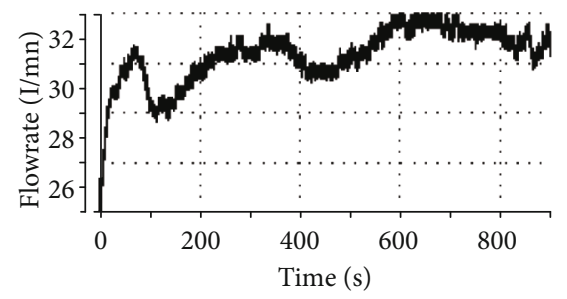

(i)

Figure 14: Experimental results of SVPWPS under constant temperature and arbitrary scenario for insolation: (a) evolution of the insolation variation; (b) evolution of the duty cycle; (c) evolution of input and optimal powers; (d) movement of MPP versus the optimum voltage; (e) a zoom of the movement of MPP versus the optimum voltage; (f) evolution of the input and output voltages; (g) stator voltage and pulsation response; (h) stator current response; (i) flow rate evolution. 


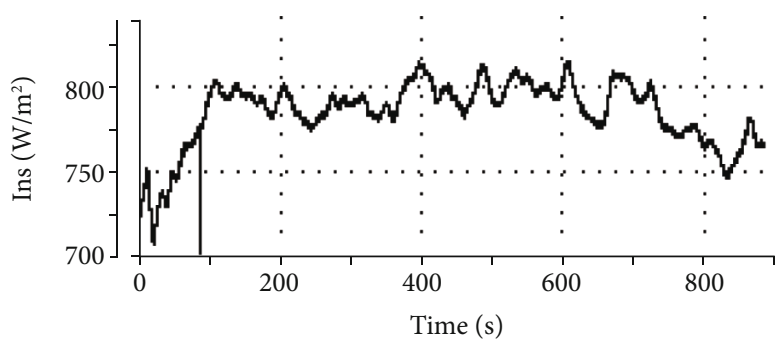

(a)



\#1:1 Vpv (Model Root/Vpv)

\#1:2 Vpv_opt (Model Root/Vpv_opt)

\#1:3 Vdc (Labels/Vdc)

(c)

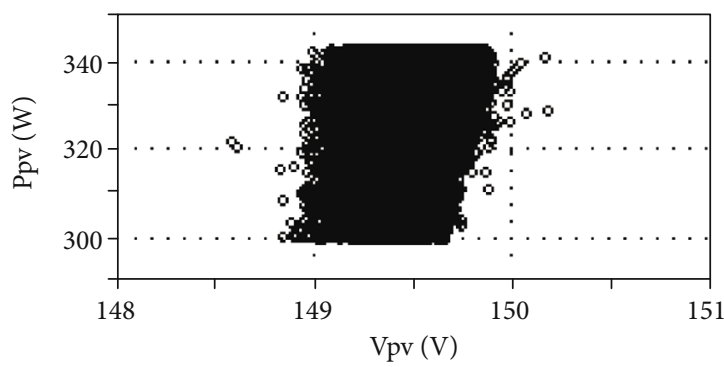

(e)

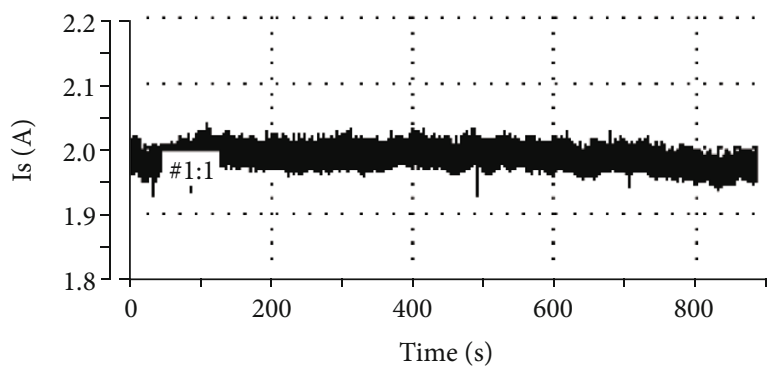

\#1:1 Is (Labels/Is)

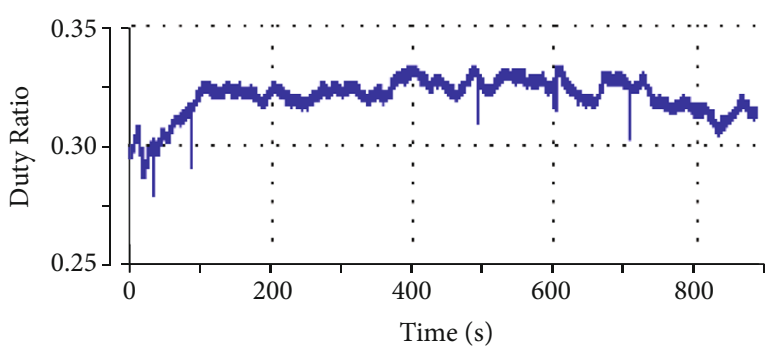

(b)

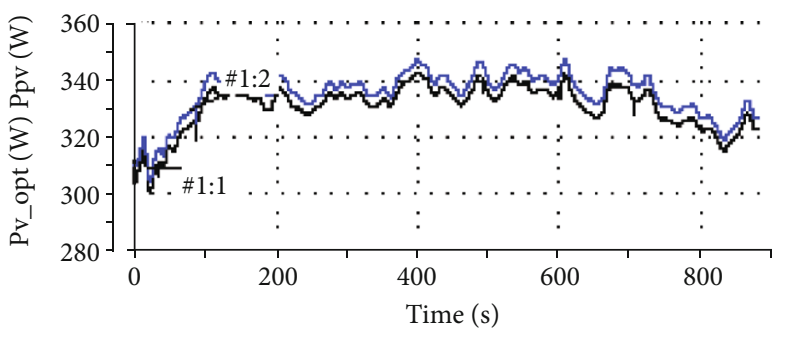

\#1:1 Ppv (Model Root/Ppv)

\#1:2 Ppv_opt (Model Root/Ppv_opt)

(d)

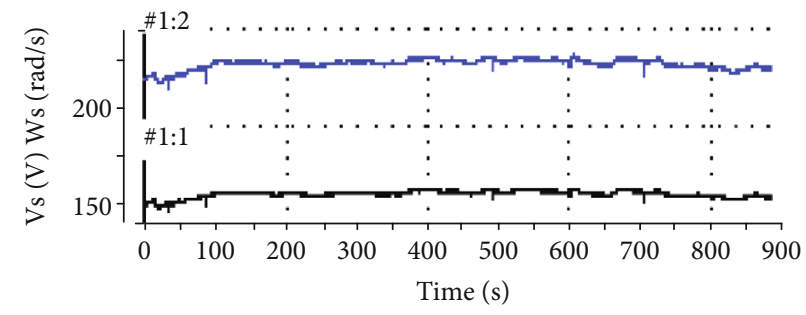

\#1:1 Vs (Labels/Vs)

\#1:2 Ws (Labels/Ws)

(f)

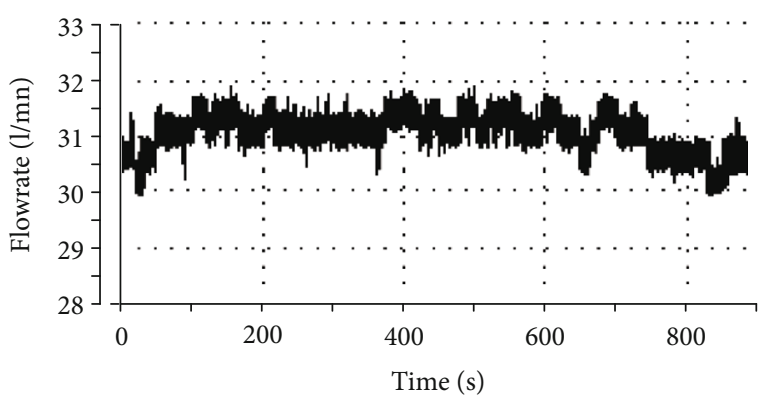

(h)

FIGURE 15: Experimental results of SVPWPS under constant temperature and insolation: (a) evolution of the insolation variation; (b) evolution of the duty cycle; (c) evolution of the input and output voltages; (d) evolution of PV powers; (e) a movement of MPP versus the optimum voltage; (f) stator voltage and pulsation response; (g) stator current response; (h) flow rate evolution.

contrary, by the proposed solution, case of the first and second test, the oscillation of the voltage does not exceed $2.5 \mathrm{~V}$ corresponding to $1.6 \%$ with respect to the requested optimal voltage. This improvement causes a better flow rate because it increases significantly with the maximum power extracted. Table 5 shows the comparison results of the proposed tech- nique with other classical MPPT techniques. The comparison is done based on previous work [23, 42-44]. And the evaluation based on experimental results shows the superior performance of the proposed algorithm over other techniques in terms of efficiency, tracking speed and accuracy, complexity of algorithm, steady-state oscillation around the 




(a)

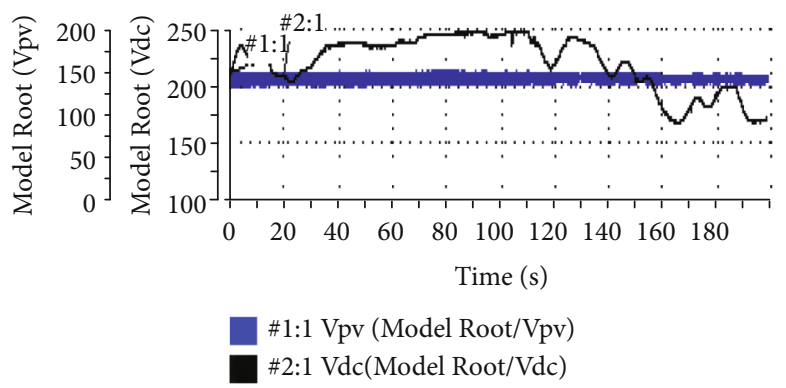

(c)

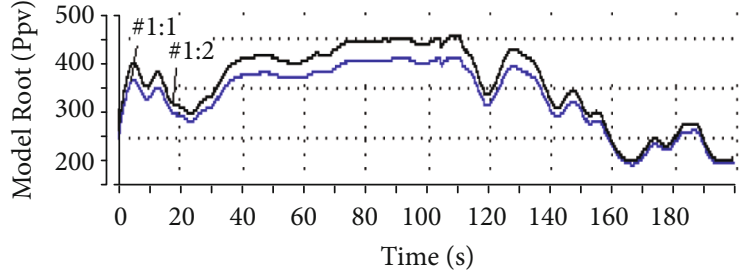

\#1:1 Pdc (Model Root/Pdc)

\#1:2 Ppv (Model Root/Ppv)

(b)

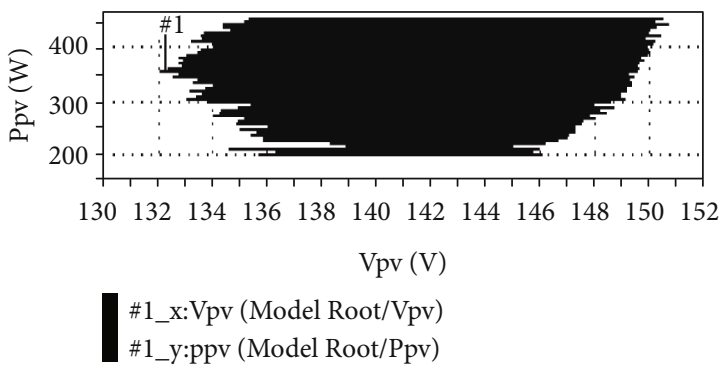

(d)

FIGURE 16: Experimental results of SVPWPS for new reference voltage and arbitrary scenario for insolation: (a) evolution of the insolation and the efficiency of the DC/DC inverter; (b) variation of the input and output powers; (c) evolution of the input and output voltage of the boost converter; (d) movement of MPP versus the PV array voltage.

TABLE 5: Experimental comparison between the proposed improved-FOCV and MPPT techniques based on classical algorithms.

\begin{tabular}{|c|c|c|c|c|c|c|}
\hline Technique & Proposed & $\mathrm{P} \& \mathrm{O}$ & IC & FOCV & $\mathrm{CV}$ & SCC \\
\hline Sensed parameters & Temperature & $\begin{array}{c}\text { Voltage and } \\
\text { current }\end{array}$ & $\begin{array}{c}\text { Voltage and } \\
\text { current }\end{array}$ & Voltage & Voltage & Current \\
\hline Tracking speed & Fast & Slow & Slow & Slow & Slow & Slow \\
\hline Tracking accuracy & High & Medium & Medium & Slow & Slow & Medium \\
\hline Control strategy & $\begin{array}{l}\text { Indirect } \\
\text { control }\end{array}$ & Direct control & Direct control & $\begin{array}{l}\text { Indirect } \\
\text { control }\end{array}$ & $\begin{array}{l}\text { Indirect } \\
\text { control }\end{array}$ & $\begin{array}{l}\text { Indirect } \\
\text { control }\end{array}$ \\
\hline Complexity level & Simple & Medium & Complex & Simple & Simple & Simple \\
\hline Cost & Inexpensive & Affordable & Expensive & Inexpensive & Inexpensive & Inexpensive \\
\hline Response to load variation & Faster & Fast & Fast & Slow & Slow & Slow \\
\hline Steady-state oscillation & Small & Large & Small & Large & Large & Medium \\
\hline Efficiency & $99 \%$ & $97.8 \%$ & $98.5 \%$ & $92.4 \%$ & $72.8 \%$ & $93.4 \%$ \\
\hline
\end{tabular}

MPP, cost, sensors needed for implementation, and response to the load variation.

\section{Performances during Start-Stop Acquisition}

To test the performance of the whole system, a series of measurements are saved at the start and stop of the drive under constant insolation and temperature of $825 \mathrm{~W} / \mathrm{m}^{2}$ and $24^{\circ} \mathrm{C}$, respectively. The MPP is tracked immediately after the starting motor. Subsequently, the measurement of PV voltage $\left(V_{\mathrm{pv}}\right)$, DC bus voltage $\left(V_{\mathrm{dc}}\right)$, insolation, and global efficiency of the PV system is made and presented in Figure 17. This figure demonstrates that the boost converter operates appropriately and quickly to extract the maximum power accord- ing to the improved FOCV MPPT technique adopted and that the global efficiency of the system depending on the available and extracted powers in this condition is $97 \%$. Furthermore, electromagnetic torque $\left(C_{e m}\right)$, flow rate $(Q)$, and stator current module $\left(I_{s}\right)$ are observed for IM coupled with a centrifugal pump (Figure 18). The electrical variables of the motor $\left(C_{e m}\right)$ and $\left(I_{s}\right)$ are well controlled by the scalar control, and therefore, the flow rate is also controlled.

The actions on the PV voltage are carried out for two instants, specifically start at 1.5 seconds and stop at $13.5 \mathrm{sec}-$ onds. The performance of the drive system on starting is adequate, and all parameters reach their equilibrium values directly after starting. Thus, establishing the steady state takes a response time of about one second. It is perceived 


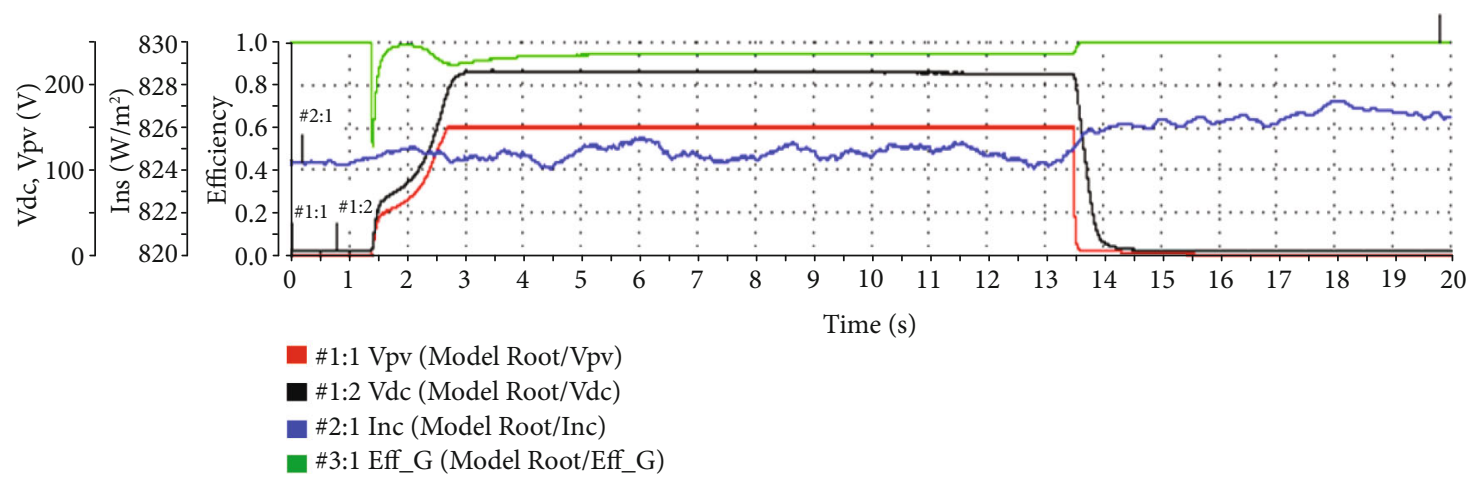

FIGURE 17: Voltage characteristics.

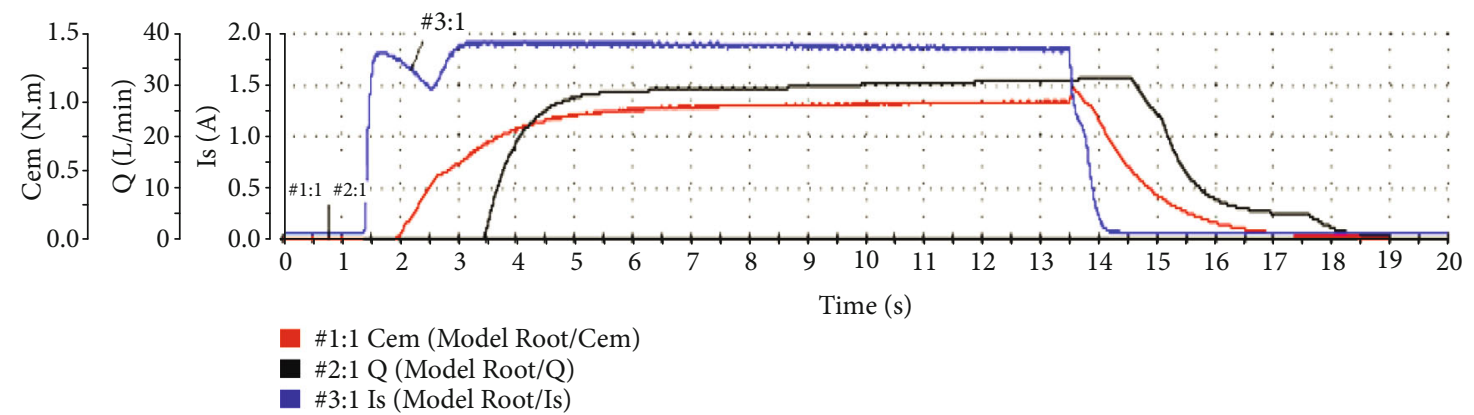

Figure 18: Pump characteristics.

that the source voltage $\left(V_{\mathrm{pv}}\right)$ is well adjusted to its optimal value of $150 \mathrm{~V}$. The available and extracted powers are practical closes that elucidate an efficiency of about $97 \%$.

For the direct starting of the IM without control, the motor's slip is unity at the time of starting, and the starting stator current is very high, which causes heating problems and a risk of damaging the system. Using the proposed solution, the three-phase inverter is switched off initially because the MPPT algorithm is not running. After starting, the DCbus voltage is controlled, and the safe starting of IM is obtained by eliminating the possibility of peak current, and the system operates at the MPP. By stopping the simulation of the control MPPT algorithm, the system returns to its initial condition. The flow rate follows the actions of electromagnetic torque and stator current of the IM.

5.1. Steady-State Test. To study the system's performance in a steady-state, a recording is made for 20 seconds at constant insulation and temperature. The characterisation of insolations, $V_{\mathrm{pv}}, V_{\mathrm{dc}}$, and efficiency is presented in Figure 19. The boost converter steps up the output voltage to $210 \mathrm{~V}$ by action on $D$ 's duty cycle to guarantee operation at the MPP. Also, power response to the system specifically $P_{\mathrm{pv}}$ and $P_{\mathrm{dc}}$ is presented in Figure 20. These powers are at maximum values, and the difference is due to semiconductor losses and the use of measurement sensors. Further, Figure 21 displays the response of $C_{e m}, Q$, and $I_{s}$. These variables are constant in this case because the insolation, the temperature, and the load are constant. From the wave- forms, it is observed that the PV voltage is settled at MPP voltage, and the variation between input and output power is minimal, which is confirmed by the efficiency of $97 \%$. Moreover, the electromagnetic torque of $1 \mathrm{Nm}$, the flow rate of $34 \mathrm{l} / \mathrm{mn}$, and the stator current module of $1.8 \mathrm{~A}$ are obtained.

Furthermore, the direct and quadratic components $I_{d s}$ and $I_{q s}$ of the stator current $I_{s}$ and PV current $I_{\mathrm{pv}}$ are extracted from the above images and demonstrated in Figure 22. These results are performed in the steady-state condition and ensure that the system is functioning correctly. A Concordia transformation is applied to obtain the current components $I_{d s}$ and $I_{q s}$. The current $I_{\mathrm{pv}}$ shows that the boost converter is operated in CCM operation. From the image, it is observed that the PV current is around $2 \mathrm{~A}$, and the direct and quadratic current components are sinusoidal with $90^{\circ} \mathrm{C}$ apart from each other.

The scalar control technique controls the steady-state performance of the proposed system. The obtained results prove that this state attained high performance with a flat torque profile, tiny ripples in inductor current, and pure sinusoidal motor current. It shows that the SVPWM technique used to drive the VSI is more convenient than other PWM techniques. Also, scalar control avoids using current sensors for its implementation, unlike FOC and DTC techniques, and therefore, it acts as a simple and inexpensive structure. On the other hand, with the proposed MPPT technique in steady state, the maximum power is extracted, and the flow rate is at the maximum level. 


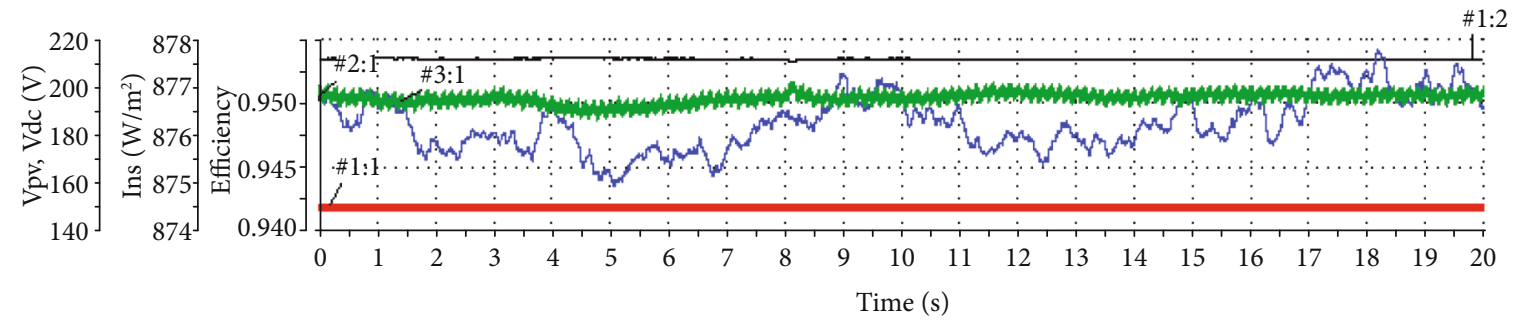

\#1:1 Vpv (Model Root/Vpv)

\#1:2 Vdc (Model Root/Vdc)

\#2:1 Inc (Model Root/Inc)

—3:1 Eff_G (Model Root/Eff_G)

FIGURE 19: Steady-state voltage characteristics.

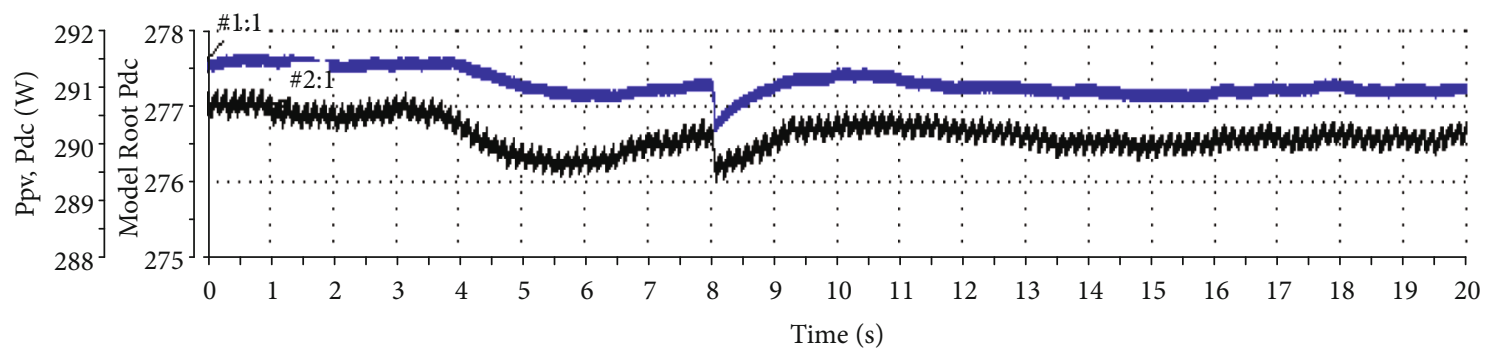

\#1:1 Ppv (Model Root/Ppv)

\#2:1 Pdc (Model Root/Pdc)

FIGURE 20: Steady-state power characteristics.

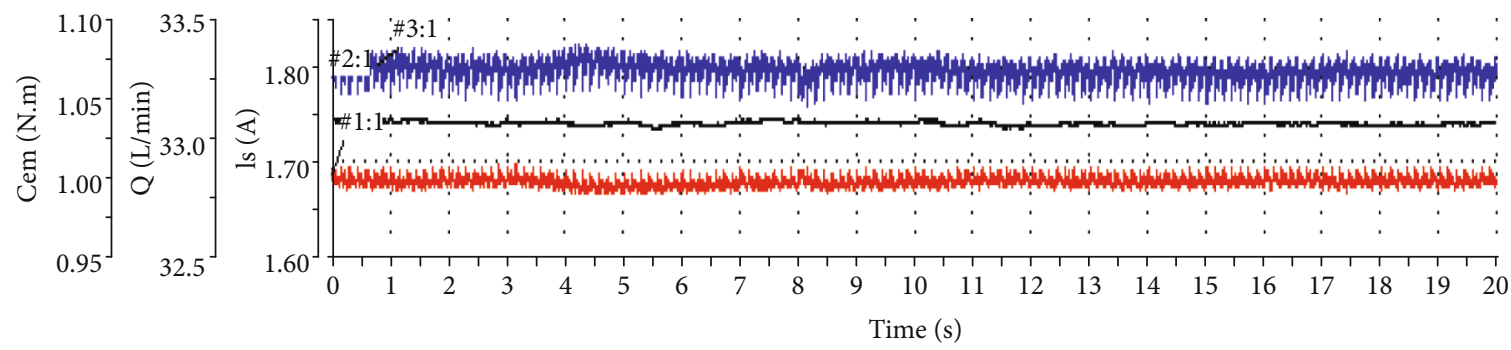

\#1:1 Cem (Model Root/Cem)

\#2:1 Q (Model Root/Q)

— \#3:1 Is (Model Root/Is)

FIGURE 21: Steady-state pump characteristics.

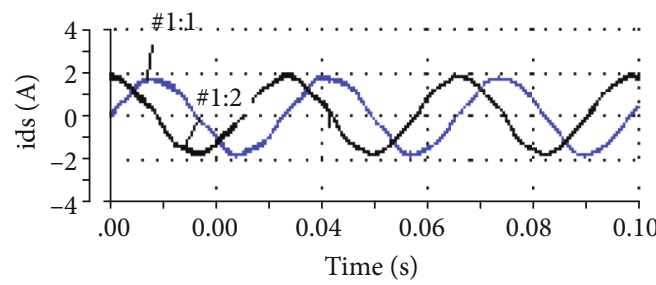

\#1:1 ids (Labels/ids)

\#1:2 iqs (Labels/iqs)

(a)

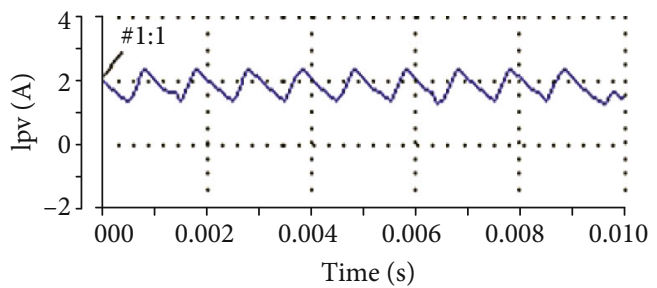

\#1:1 lpv (Labels/lpv)

(b)

Figure 22: Currents response: (a) zoom on direct and quadratic components $I_{d s}$ and $I_{q s}$; (b) zoom on PV array current $I_{\mathrm{pv}}$. 
Consolidating these results, the main objective of this application is to investigate an efficient, easy-to-use, highperformance SPVWPS to allow the use of electricity in rural areas disconnected from the grid or in the case of a significant energy deficit that negatively affects agricultural production. To accelerate the design process and prove the ability of the proposed algorithm, an RCP methodology is applied. A set of electronic circuits are used in the laboratory to complete this solution: sensors (temperature, insolation, voltages, and currents), TTL-CMOS circuit driver for boost DC/DC, VSI, and flow meter circuit. The key benefits of RCP are increasing rapidly because manual programming has more disadvantages; the cost is reduced compared to the amount of work that would be required to perform a similar test setup from scratch. The control function can be managed in an easy and fast way, and the evaluation is instantaneous. Lastly, the efficiency of RCP is proven, which clearly shows its effectiveness from the test above. Specifically, the withdrawn power from the PV array is at its maximum value for all variations in climatic conditions.

\section{Conclusions}

The design and implementation of an off-grid SPVWPS are proposed in this work. This system is operated using an RCP platform that integrates an improved FOCV MPPT method and closed-loop scalar control. The mathematical models of the PV array, DC-DC boost converter, three-phase VSI, and IM coupled to centrifugal pump are described in detail. The following conclusions are made:

(i) The proposed scalar control allows controlling the induction motor according to the maximum power available in the PV panel and eliminates extra sensors for speed and current measurement

(ii) An improved fractional open circuit voltage MPPT method enhances the efficiency of the conventional algorithm

(iii) The experimental results displayed the accurate performance of the proposed solution in terms of global efficiency optimization of the SPVWPS, better stability for the exact parameters of the system under start and stop acquisition, and water discharge rates in the steady-state operation

(iv) The effectiveness of the chosen MPPT method for the optimization of the photovoltaic pumping system is tested, and correct steady-state and dynamic operation, including severe solar insolation variations, have been obtained for the whole system

Therefore, the proposed system can be potentially used to design an off-grid solar photovoltaic water pumping system.

\section{Data Availability}

The data used to support the findings of this study are included in the article.

\section{Conflicts of Interest}

The authors declare that there is no conflict of interest regarding the publication of this article.

\section{References}

[1] F. Johnsson, J. Kjärstad, and J. Rootzén, "The threat to climate change mitigation posed by the abundance of fossil fuels," Climate Policy, vol. 19, no. 2, pp. 258-274, 2019.

[2] R. Madurai Elavarasan, L. Selvamanohar, K. Raju et al., "A holistic review of the present and future drivers of the renewable energy mix in Maharashtra, state of India," Sustainability, vol. 12, no. 16, p. 6596, 2020.

[3] A. K. Mishra and B. Singh, "Design of solar-powered agriculture pump using new configuration of dual-output buck-boost converter," IET Renewable Power Generation, vol. 12, no. 14, pp. 1640-1650, 2018.

[4] C. Ramulu, P. Sanjeevikumar, R. Karampuri, S. Jain, A. H. Ertas, and V. Fedak, "A solar PV water pumping solution using a three-level cascaded inverter connected induction motor drive," Engineering Science and Technology, an International Journal, vol. 19, no. 4, pp. 1731-1741, 2016.

[5] R. Antonello, M. Carraro, A. Costabeber, F. Tinazzi, and M. Zigliotto, "Energy-efficient autonomous solar waterpumping system for permanent-magnet synchronous motors," IEEE Transactions on Industrial Electronics, vol. 64, no. 1, pp. 43-51, 2017.

[6] U. Sharma, B. Singh, and S. Kumar, "Intelligent grid interfaced solar water pumping system," IET Renewable Power Generation, vol. 11, no. 5, pp. 614-624, 2017.

[7] S. Murshid and B. Singh, "Implementation of PMSM drive for a solar water pumping system," IEEE Transactions on Industry Applications, vol. 55, no. 5, pp. 4956-4964, 2019.

[8] M. Errouha, A. Derouich, B. Nahid-Mobarakeh, S. Motahhir, and A. El Ghzizal, "Improvement control of photovoltaic based water pumping system without energy storage," Solar Energy, vol. 190, pp. 319-328, 2019.

[9] A. K. Mishra and B. Singh, "Grid interactive single-stage solar powered water pumping system utilizing improved control technique," IEEE Transactions on Sustainable Energy, vol. 11, no. 1, pp. 304-314, 2020.

[10] C. Liu and Y. Luo, "Overview of advanced control strategies for electric machines," Chinese Journal of Electrical Engineering, vol. 3, 2017.

[11] O. Ellabban, J. Van Mierlo, and P. Lataire, "A comparative study of different control techniques for an induction motor fed by a Z-source inverter for electric vehicles," in 2011 International Conference on Power Engineering, Energy and Electrical Drives, pp. 1-7, Malaga, Spain, 2011.

[12] I. Boldea, "Control issues in adjustable speed drives," IEEE Industrial Electronics Magaziner, vol. 2, no. 3, pp. 32-50, 2008.

[13] T. H. Dos Santos, A. Goedtel, S. A. O. Da Silva, and M. Suetake, "Scalar control of an induction motor using a neural sensorless technique," Electric Power Systems Research, vol. 108, pp. 322 330, 2014.

[14] Chun-Chieh Wang and Chih-Hsing Fang, "Sensorless scalarcontrolled induction motor drives with modified flux observer," IEEE Transactions on Energy Conversion, vol. 18, no. 2, pp. 181-186, 2003. 
[15] A. R. Jordehi, "Maximum power point tracking in photovoltaic (PV) systems: a review of different approaches," Renewable and Sustainable Energy Reviews, vol. 65, pp. 1127-1138, 2016.

[16] A. K. Podder, A. K. Das, E. Hossain et al., "Integrated Modeling and Feasibility Analysis of a Rooftop Photovoltaic Systems for an Academic Building in Bangladesh," International Journal of Low-Carbon Technologies, 2021.

[17] D. Singh, R. Chaudhary, and A. Karthick, "Review on the progress of building-applied/integrated photovoltaic system," Environmental Science and Pollution Research, vol. 28, no. 35, pp. 47689-47724, 2021.

[18] C. Pazhanimuthu, I. Baranilingesan, and A. Karthick, “An improved control algorithm for series hybrid active power filter based on SOGI-PLL under dynamic load conditions," Solid State Communications, vol. 333, 2021.

[19] N. M. Kumar, M. Samykano, and A. Karthick, "Energy loss analysis of a large scale BIPV system for university buildings in tropical weather conditions: a partial and cumulative performance ratio approach," Case Studies in Thermal Engineering, vol. 25, 2021.

[20] O. Singh and S. K. Gupta, "A review on recent MPPT techniques for photovoltaic system," in 2018 IEEMA Engineer Infinite Conference (eTechNxT), pp. 1-6, New Delhi, India, 2018.

[21] V. Salas, E. Olías, A. Barrado, and A. Lázaro, "Review of the maximum power point tracking algorithms for stand-alone photovoltaic systems," Solar Energy Materials and Solar Cells, vol. 90, no. 11, pp. 1555-1578, 2006.

[22] A. R. Prasad, R. Shankar, C. K. Patil, A. Karthick, A. Kumar, and R. Rahim, "Performance enhancement of solar photovoltaic system for roof top garden," Environmental Science and Pollution Research, vol. 28, no. 36, pp. 50017-50027, 2021.

[23] R. Kabilan, V. Chandran, J. Yogapriya et al., "Short-term power prediction of building integrated photovoltaic (BIPV) system based on machine learning algorithms," International Journal of Photoenergy, vol. 2021, 11 pages, 2021.

[24] N. Y. Jayalakshmi, R. Shankar, U. Subramaniam et al., "Novel multi-time scale deep learning algorithm for solar irradiance forecasting," Energies, vol. 14, no. 9, p. 2404, 2021.

[25] A. Hmidet, N. Rebei, and O. Hasnaoui, "Experimental studies and performance evaluation of MPPT control strategies for solar-powered water pumps," in 2015 Tenth International Conference on Ecological Vehicles and Renewable Energies (EVER),, pp. 1-12, Monte Carlo, Monaco, 2015.

[26] V. S. Chandrika, M. M. Thalib, A. Karthick et al., "Performance assessment of free standing and building integrated grid connected photovoltaic system for southern part of India," Building Services Engineering Research and Technology, vol. 42, no. 2, pp. 237-248, 2021.

[27] M. A. Eltawil and Z. Zhao, "MPPT techniques for photovoltaic applications," Renewable and Sustainable Energy Reviews, vol. 25, pp. 793-813, 2013.

[28] S. Kıvrak, T. Özer, and Y. Oğuz, "Design and Implementation of dspic33fj32mc204 Microcontroller-Based Asynchronous Motor Voltage/Frequency Speed Control Circuit for the Ventilation Systems of Vehicles," Measurement and Control, vol. 52, no. 7-8, pp. 1039-1047, 2019.

[29] L. Jun, G. Ying-Qing, and W. Hai-Quan, "Rapid prototyping real-time simulation platform for digital electronic engine control," in 2008 2nd International Symposium on Systems and Control in Aerospace and Astronautics, pp. 1-5, Shenzhen, China, 2008.
[30] A. Hmidet and O. Boubaker, "Real-Time Low-Cost Speed Monitoring and Control of Three-Phase Induction Motor via a Voltage/Frequency Control Approach," Mathematical Problems in Engineering, vol. 2020, Article ID 6913813, 14 pages, 2020.

[31] J. Aravena, D. Carrasco, M. Diaz et al., "Design and implementation of a low-cost real-time control platform for power electronics applications," Energies, vol. 13, no. 6, 2020.

[32] S. Wendel, A. Geiger, E. Liegmann et al., "UltraZohm - a powerful real-time computation platform for MPC and multi-level inverters," in 2019 IEEE International Symposium on Predictive Control of Electrical Drives and Power Electronics (PRECEDE), pp. 1-6, Quanzhou, China, 2019.

[33] A. Hmidet, R. Dhifaoui, and O. Hasnaoui, "Development, implementation and experimentation on a dSPACE DS1104 of a direct voltage control scheme," Journal of Power Electronics, vol. 10, no. 5, pp. 468-476, 2010.

[34] A. R. Jordehi, "Parameter estimation of solar photovoltaic (PV) cells: a review," Renewable and Sustainable Energy Reviews, vol. 61, pp. 354-371, 2016.

[35] P. Sahu, D. Verma, and S. Nema, "Physical design and modelling of boost converter for maximum power point tracking in solar PV systems," in 2016 International Conference on Electrical Power and Energy Systems (ICEPES), pp. 10-15, Bhopal, India, 2016.

[36] M. Lasheen, A. K. Abdel Rahman, M. Abdel-Salam, and S. Ookawara, "Adaptive reference voltage-based MPPT technique for PV applications," IET Renewable Power Generation, vol. 11, no. 5, pp. 715-722, 2017.

[37] B. Singh, U. Sharma, and S. Kumar, "Standalone photovoltaic water pumping system using induction motor drive with reduced sensors," IEEE Transactions on Industry Applications, vol. 54, no. 4, pp. 3645-3655, 2018.

[38] A. Hmidet, R. Dhifaoui, and O. Hasnaoui, "A new direct speed estimation and control of the induction machine benchmark: design and experimental validation," Mathematical Problems in Engineering, vol. 2018, Article ID 9215459, 10 pages, 2018.

[39] V. K. Arun Shankar, S. Umashankar, S. Paramasivam, and N. Hanigovszki, "A comprehensive review on energy efficiency enhancement initiatives in centrifugal pumping system," Applied Energy, vol. 181, pp. 495-513, 2016.

[40] B. Singh and S. Shukla, "Induction Motor Drive for PV Water Pumping with Reduced Sensors," IET Power Electronics, vol. 11, no. 12, pp. 1903-1913, 2018.

[41] A. Smith, S. Gadoue, M. Armstrong, and J. Finch, "Improved method for the scalar control of induction motor drives," IET Electric Power Applications, vol. 7, no. 6, pp. 487-498, 2013.

[42] L. Xu, R. Cheng, and J. Yang, "A new MPPT technique for fast and efficient tracking under fast varying solar irradiation and load resistance," International Journal of Photoenergy, vol. 2020, Article ID 6535372, 18 pages, 2020.

[43] A. Nadeem, H. A. Sher, and A. F. Murtaza, "Online fractional open-circuit voltage maximum output power algorithm for photovoltaic modules," IET Renewable Power Generation, vol. 14, no. 2, pp. 188-198, 2020.

[44] R. B. Bollipo, S. Mikkili, and P. K. Bonthagorla, "Hybrid, optimization, intelligent and classical PV MPPT techniques: review," CSEE Journal of Power and Energy Systems, vol. 7, 2020. 